University of Wollongong

Research Online

Australian Institute for Innovative Materials -

Papers

Australian Institute for Innovative Materials

$1-1-2019$

Evaluation of sterilisation methods for bio-ink components: gelatin, gelatin methacryloyl, hyaluronic acid and hyaluronic acid methacryloyl

\author{
Cathal D. O'Connell \\ University of Wollongong, cathal.oconnell@svha.org.au \\ Carmine Onofrillo \\ University of Melbourne, carmine@uow.edu.au \\ Serena Duchi \\ University of Melbourne \\ Xin Li \\ University of Melbourne \\ Yifan Zhang \\ University of Melbourne
}

See next page for additional authors

Follow this and additional works at: https://ro.uow.edu.au/aiimpapers

Part of the Engineering Commons, and the Physical Sciences and Mathematics Commons

Research Online is the open access institutional repository for the University of Wollongong. For further information contact the UOW Library: research-pubs@uow.edu.au 


\title{
Evaluation of sterilisation methods for bio-ink components: gelatin, gelatin methacryloyl, hyaluronic acid and hyaluronic acid methacryloyl
}

\begin{abstract}
Reliable and scalable sterilisation of hydrogels is critical to the clinical translation of many biofabrication approaches, such as extrusion-based 3D bioprinting of cell-laden bio-inks. However sterilisation methods can be destructive, and may have detrimental effects on the naturally-derived hydrogels that constitute much of the bio-ink palette. Determining effective sterilisation methods requires detailed analysis of the effects of sterilisation on relevant properties such as viscosity, printability and cytocompatibility. Yet there have been no studies specifically exploring the effects of sterilisation on bio-inks to date. In this work, we explored the effects of various sterilisation techniques on four of the most widely used bio-ink components: gelatin, gelatin methacryloyl, hyaluronic acid, and hyaluronic acid methacrylate. Autoclaving was the most destructive sterilisation method, producing large reductions in viscosity and in mechanical properties following crosslinking. Filter sterilisation caused some reduction in rheological properties of GelMA due to removal of higher molecular weight components, but did not affect photocrosslinking. Ethylene oxide (EtO) was the least destructive sterilisation method in terms of rheological properties for all materials, had no detrimental effect on the photocrosslinkable methacrylate/methacrylamide groups, and so was chosen for more detailed examination. In biological analyses, we found that EtO treatment successfully eradicated a bacterial challenge of E. coli, caused no decrease in viability of human mesenchyman stem cells (hMSCs), and had no effect on their rate of proliferation. Finally, we found that EtO-treated hydrogels supported encapsulated hMSCs to differentiate towards the chondrogenic lineage, and to produce new cartilage matrix. Our results bring to light various effects that sterilisation can have on bio-inks, as well as highlighting EtO sterilisation as a method which minimises degradation of properties, while still promoting biological function.

Disciplines

Engineering | Physical Sciences and Mathematics

\section{Publication Details}

O'Connell, C. D., Onofrillo, C., Duchi, S., Li, X., Zhang, Y., Tian, P., Lu, L., Trengove, A., Quigley, A., Gambhir, S., Khansari, A., Mladenovska, T., O'Connor, A., Di Bella, C., Choong, P. \& Wallace, G. G. (2019). Evaluation of sterilisation methods for bio-ink components: gelatin, gelatin methacryloyl, hyaluronic acid and hyaluronic acid methacryloyl. Biofabrication, 11 (3), 035003-1-035003-18.
\end{abstract}

\section{Authors}

Cathal D. O'Connell, Carmine Onofrillo, Serena Duchi, Xin Li, Yifan Zhang, Peilin Tian, Lanyita Lu, Anna Trengove, Anita F. Quigley, Sanjeev Gambhir, Afsaneh Khansari, Tajanka Mladenovska, Andrea O'Connor, Claudia Di Bella, Peter Choong, and Gordon G. Wallace 


\title{
Evaluation of sterilisation methods for bio-ink components: gelatin, gelatin methacryloyl, hyaluronic acid and hyaluronic acid methacryloyl
}

\author{
Authors: Cathal D. O'Connell*,,2 Carmine Onofrillo, ${ }^{1,3}$ Serena Duchi, ${ }^{1,3}$ Xin Li, ${ }^{4}$ \\ Yifan Zhang, ${ }^{4}$ Peilin Tian, ${ }^{4}$ Lanyita Lu, ${ }^{4}$ Anna Trengove, ${ }^{4}$ Anita Quigley, ${ }^{1,2}$ Sanjeev \\ Gambhir, ${ }^{2}$ Afsaneh Khansari, ${ }^{2}$ Tajanka Mladenovska, ${ }^{1,3}$ Andrea O'Connor, ${ }^{4}$ Claudia \\ Di Bella, ${ }^{3,5}$ Peter F Choong, ${ }^{3,5}$ Gordon G Wallace $^{2}$
}

${ }^{1}$ BioFab3D, Aikenhead Centre for Medical Discovery, St Vincent's Hospital, Melbourne ${ }^{2}$ ARC Centre of Excellence for Electromaterials Science, Intelligent Polymer Research Institute, Innovation Campus, University of Wollongong, NSW, Australia

${ }^{3}$ Department of Surgery, St Vincent's Hospital, University of Melbourne, Clinical Sciences Building, 29 Regent Street, 3065 Fitzroy, VIC, Australia

${ }^{4}$ Department of Biomedical Engineering, University of Melbourne, Parkville, VIC, Australia

${ }^{5}$ Department of Orthopaedics, St Vincent's Hospital, Fitzroy, Victoria, Australia

* Corresponding author

Cathal O'Connell, $\mathrm{PhD}$

BioFab3D, Aikenhead Centre for Medical Discovery, St Vincent's Hospital, Melbourne

Cathal.oconnell@svha.org.au

Lev 1, Clinical Science Building

29 Regent St, 3065 Fitzroy, VIC, Australia

Keywords

Sterilisation

3D bioprinting

Bio-ink development

Hydrogels

Photocrosslinking 


\section{Abstract ( $<300$ words)}

Reliable and scalable sterilisation of hydrogels is critical to the clinical translation of many biofabrication approaches, such as extrusion-based 3D bioprinting of cell-laden bio-inks. However sterilisation methods can be destructive, and may have detrimental effects on the naturally-derived hydrogels that constitute much of the bio-ink palette. Determining effective sterilisation methods requires detailed analysis of the effects of sterilisation on relevant properties such as viscosity, printability and cytocompatibility. Yet there have been no studies specifically exploring the effects of sterilisation on bio-inks to date.

In this work, we explored the effects of various sterilisation techniques on four of the most widely used bio-ink components: gelatin, gelatin methacryloyl, hyaluronic acid, and hyaluronic acid methacryloyl. Autoclaving was the most destructive sterilisation method, producing large reductions in viscosity and in mechanical properties following crosslinking. Filter sterilisation caused some reduction in rheological properties of GelMA due to removal of higher molecular weight components, but did not affect photocrosslinking. Ethylene oxide (EtO) was the least destructive sterilisation method in terms of rheological properties for all materials, had no detrimental effect on the photocrosslinkable methacrylate/methacrylamide groups, and so was chosen for more detailed examination. In biological analyses, we found that EtO treatment successfully eradicated a bacterial challenge of E.Coli, caused no decrease in viability of human mesenchyman stem cells (hMSCs), and had no effect on their rate of proliferation. Finally, we found that EtO-treated hydrogels supported encapsulated hMSCs to differentiate towards the chondrogenic lineage, and to produce new cartilage matrix. Our results bring to light various effects that sterilisation can have on bio-inks, as well as highlighting EtO sterilisation as a method which minimises degradation of properties, while still promoting biological function. 


\section{Introduction}

Reliable and effective sterilisation of hydrogel based bio-inks is paramount for translation of 3D bioprinting to clinical practice. Efficient sterilisation is crucial to minimize the incidence of implant-related infections, which are a major concern in health care. ${ }^{1}$ Demonstration of compliance with sterility standards (such as ISO 11737: Sterilisation of medical devices) may also be required by regulators. However the logistical requirements for sterilising materials for biofabrication and bioprinting are different than those for implants. While medical devices or biomaterial scaffolds can typically be sterilised in a terminal stage, immediately prior to implantation or packaging, ${ }^{2,3}$ bio-inks for live cell printing must be sterilised much earlier: i.e. at least prior to the encapsulation of cells. This necessitates a fresh examination of the sterilisation workflow in the context of biofabrication.

Despite the importance of sterilisation, its effects on the properties of hydrogels remains understudied, ${ }^{1}$ and most particularly in the bioprinting space. To our knowledge, there have been no studies to date which address the sterilisation of bio-inks for 3D bioprinting. This is surprising considering the recent explosion in research on bio-ink development. The extant hydrogel sterilisation literature is not sufficient because it typically does not address certain properties of key importance for bio-inks, such as rheology, printability and the mechanical properties following crosslinking. On top of this, the past decade has seen particular classes of photocrosslinkable bio-ink materials, such as methacrylate/methacrylamide functionalised hydrogels, rise in prominence in the field of biofabrication. Again, we have not uncovered any studies which address the effect of sterilisation methods on this emerging family of materials.

Sterilisation will unavoidably cause some changes to materials. The purpose of sterilisation is to remove or inactivate microbial contaminants that may be present, typically by denaturing proteins, disrupting cellular membranes or enzyme systems, or destroying their nucleic acid bases. Each of these approaches has the potential to alter the biomaterial being sterilised, which may compromise performance. Hydrogels and soft biomaterials are especially susceptible to such collateral damage, being highly sensitive to common sterilising agents, such as heat and radiation. ${ }^{1}$ Sterilisation can lead to degradation and/or decomposition of materials, discoloration, embrittlement, odor generation, and can promote further crosslinking or induce toxic effects. ${ }^{1}$ There is thus a need to determine sterilisation processes which minimises hydrogel deterioration, while still guaranteeing sterility. Furthermore, any unavoidable effects 
of sterilisation on relevant properties should be documented, and taken into account during the bio-ink formulation stage.

The critical characteristics of bio-inks encompass rheological and mechanical properties, biocompatibility, presence of cell-adhesion sites and degradability among others, and all of these properties must be retained following sterilisation. For example, to maintain printability, bio-inks require rheological properties such as a high yield stress, high viscosity and strong shear thinning behaviour ${ }^{4}$. Some hydrogels, such as gelatin and hyaluronic acid, are commonly added to bio-inks to modify rheological properties to favour printing. ${ }^{5-9}$ Sterilisation should not overly disrupt these critical rheological characteristics.

Besides having suitable rheological properties, bio-inks are typically designed to form 'bioscaffold' environments for cell encapsulation in 3D. To maintain shape fidelity, bio-scaffolds must be structurally stable under physiological conditions. Common strategies to achieve this include the formation of crosslinked hydrogel networks, using photoinduced free-radical polymerization $^{10-12}$ and enzymatic-based crosslinking ${ }^{13,14}$. In recent years, the strategy to functionalise naturally derived hydrogels with photocrosslinkable groups has come to the fore, producing materials such as gelatin methacryoyl (GelMA) $)^{10,15-20}$ and hyaluronic acid methacryloyl (HAMA). ${ }^{8,16,21-24}$ These materials have potential applications across a wide range of tissues, depending on how their mechanical properties are tailored. ${ }^{8,12,20}$ Thus, sterilisation processes applied to these materials must also be screened for their effects on hydrogel crosslinking.

Methods previously reported for sterilising bioink materials include UV irradiation ${ }^{25-28}$, autoclaving $^{6,29,30}$ (especially for gelatin), repeated heating, ${ }^{5}$ and filter-sterilisation ${ }^{15,17}$ (especially for GelMA). Ethylene oxide (EtO) gas $^{31,32}$ and gamma-irradiation ${ }^{21}$ have also been used, although more rarely. Most often, the sterilisation method is not reported.

In this work, we examined the effects of various sterilisation methods on four of the most common hydrogels used to formulate bio-inks for 3D bioprinting: gelatin, ${ }^{5-7,9}$ GelMA, ${ }^{10,15-20}$ hyaluronic acid (HA), ${ }^{8,21}$ and HAMA. ${ }^{16,22-24}$ We decided to characterise each of these hydrogels individually since different combinations of these materials can be used to formulate any number of bio-inks targeting particular tissues. The paper is organised broadly in two sections. The first half is an exploration of the effect of various sterilisation methods on the physical properties (rheological and mechanical) of these four bio-ink components. Here we evaluated the effects of both autoclaving and EtO-treatment for all four hydrogels, as well as sterile filtration for GelMA (the other materials being unable to be filtered). Through these analyses, ethylene oxide treatment (EtO) emerged as the least destructive sterilisation method 
in terms of the physical and chemical properties tested, and so was chosen for more detailed examination. The second half of the paper focuses on biological characterisation of EtOsterilised materials. After assessing the efficacy of EtO-treatment in eradicating a bacterial challenge, we assayed the behaviour of human mesenchymal stem cells (hMSCs) encapsulated within EtO-treated materials in terms of their viability, proliferation and differentiation.

Our results bring to light the various effects that sterilisation can have on bio-inks, as well as highlighting EtO treatment as a method which minimises degradation of properties, while still promoting biological function.

\section{Methods}

\subsection{Materials}

Gelatin type A from porcine skin ( 300 Bloom) was sourced from PB Leiner and hyaluronic acid sodium salt from Streptococcus equi (mol wt. 1.6 2.0 x 10 $10^{6} \mathrm{Da}$ ) was obtained from Boading Faithful Industries Co. Ltd. Gelatin-methacryloyl (GelMA) and hyaluronic acid methacrylate (HAMA) were synthesized as described previously. ${ }^{24}$ The degree of functionalization for GelMA (70\%) and HAMA (20\% $\pm 3 \%)$ was quantified by ${ }^{1} \mathrm{H}-\mathrm{NMR}$ in $\mathrm{D}_{2} \mathrm{O} .{ }^{33}$ The freeze-dried products were stored at $-80{ }^{\circ} \mathrm{C}$ prior to use.

\subsection{Materials preparation and sterilisation}

\subsubsection{Autoclave sterilisation}

The materials were dissolved to a final concentration of $100 \mathrm{mg} \mathrm{ml}^{-1}$ (GelMA or gelatin) and $40 \mathrm{mg} \mathrm{ml}^{-1}$ (HA or HAMA) in sterile PBS (Sigma-Aldrich). Autoclaving was performed in a Tomy Autoclave SX 500E for 20 minutes at $121^{\circ} \mathrm{C}$.

\subsubsection{EtO sterilisation}

All materials were EtO sterilised in powder form. Powders were aliqoted into $50 \mathrm{ml}$ centrifuge tubes with vented caps, $0.2 \mu \mathrm{m}$ pore size (Corning $50 \mathrm{ml}$ mini bioreactor, Sigma-Aldrich, cat. no. CLS431720). Aliquots were held in a humid environment at room temperature for $4 \mathrm{hrs}$ to ensure any bacterial spores were not in a dessicated state. (Some bacterial spores can become resistant to ethylene oxide sterilisation if dessicated). ${ }^{34}$ Alternatively, a drop of water can be added to each tube to hydrate lyophlized hydrogels. 
EtO sterilisation was performed in an Anprolene EtO sterilizer (Anderson Sterilizers Inc., USA) at room temperature $\left(23^{\circ} \mathrm{C}\right)$ for a 12 -hour cycle, followed by a further 12-hour purge to flush the materials/plasticware of residual EtO. Materials were prepared according to the manufacturer's instructions using an Anprolene ampoule, plastic liner bag and sterilizer container. The Anprolene system is designed to allow diffusion of the EtO gas at a rate slow enough to sterilize the contents. Each ampoule releases approximately $3800 \mathrm{mg} \mathrm{EtO}$ at room temperature and atmospheric pressure, resulting in a minimum peak concentration within the liner bag of $500 \mathrm{mg} / 1000 \mathrm{~cm}^{3}$. For consistent results it is important to load the sterilizer with similar quantities of plasticware per cycle, since plastics absorb EtO during the cycle and reduce the effective concentration. An EtO dosimeter chemical indicator (Anderson), placed inside a vessel/package identical to those containing hydrogel, was used to confirm adequate and consistent dosing of EtO for each cycle.

\subsubsection{Filter sterilisation}

GelMA was dissolved in MilliQ water at a concentration of $1.5 \% \mathrm{w} / \mathrm{v}$ GelMA, and filtered at $37^{\circ} \mathrm{C}$ in a class II biological safety cabinet using sterile $0.22-\mu \mathrm{m}$ syringe filter units with a polyethersulfone (PES) membrane. PVDF and glass membranes were also trialled, but these could filter very little material before clogging. Filtered GelMA solutions were freeze-dried (Martin Christ Alpha 1-2 LDplus) in pre-weighed tubes equipped with vent caps (Mini Bioreactor Centrifuge Tube, Corning) for 4 days, or until the mass tubes had stabilised, then stored in weighed aliqots in closed vessels at $-80^{\circ} \mathrm{C}$ until needed.

\subsection{Rheology}

All rheology experiments were performed on a Physica MCR 302 Rheometer (Anton Paar) in a cone-plate geometry $\left(15 \mathrm{~mm}\right.$ diameter with a cone angle of $1^{\circ}$, and a truncation of $\left.31 \mu \mathrm{m}\right)$ at room temperature $\left(23^{\circ} \mathrm{C}\right)$. For flow experiments, the shear rate was ramped up from 0.001 to $100 \mathrm{~s}^{-1}$ over 200s. The yield stress was measured using the viscosity maximum method, i.e. the stress at maximum viscosity.

\subsection{In-situ photorheology}

In-situ photorheology experiments were performed as previously described. ${ }^{12}$ Briefly, oscillatory measurements were performed at $1 \%$ strain and an angular frequency of $10 \mathrm{rad} \mathrm{s}^{-1}$. For in situ UV curing, 365nm light from the UV light source (Omnicure LX400+, Lumen Dynamix LDGI) was used to illuminate the underside of the sample through a quartz crystal stage. The UV intensity $\left(100 \mathrm{~mW} / \mathrm{cm}_{2}\right)$ was measured at the sample using a UV meter (Omnicure) before each experiment. 


\subsection{Gel permeation chromatography (GPC)}

All GPC analysis was performed on Shimadzu, LC-20 system comprising an isocratic pump, an autosampler with variable injection volume, and a refractive index detector using two columns connected in series. The first column was an ultrahydrogel 500,10 $\mu \mathrm{m}, 7.8 \times 300 \mathrm{~mm}$, mol.wt. range $10 \mathrm{~K}-400 \mathrm{~K}$ from Waters (part no. WAT011530) and second column was ultrhydrogel 250, $6 \mu \mathrm{m}, 7.8 \times 300 \mathrm{~mm}$, mol.wt. range $1 \mathrm{~K}-80 \mathrm{~K}$ from Waters (part no. WAT011525). Phosphate buffer with $0.2 \mathrm{M} \mathrm{NaCl}$ and $0.5 \%$ sodium docecyl sulphate was used as mobile phase and the $\mathrm{pH}$ was 5.5. A flow rate of $0.6 \mathrm{~mL} / \mathrm{min}$ was maintained. Temperature of the column was kept at $35^{\circ} \mathrm{C}$. The concentration of GelMA samples for injection was $5 \mathrm{mg} /$ $2 \mathrm{ml}$ and injection volume was $100 \mu \mathrm{L} . \mathrm{n}=1$ measurement for each GelMA sample. Poly (styrene sulphonate) sodium salt standards $(6 \mathrm{~K}, 17 \mathrm{~K}, 30 \mathrm{~K}, 74 \mathrm{~K}, 200 \mathrm{~K}, 400 \mathrm{~K})$ were used for the calibration curve.

\subsection{Enzymatic crosslinking and mechanical testing}

Microbial transglutaminase (TG) powder (Activa, Melbourne Food Depot) was dissolved in DPBS, filter sterilized and added to gelatin solutions to make a final concentration of $10 \%$ gelatin w/v and $2 \%$ TG. After thorough mixing, this solution was extruded into PDMS moulds, covered with glass coverslips achieve flat surfaces, and incubated in a humidified environment for $24 \mathrm{hr}$ to allow enzymatic crosslinking to reach completion. After crosslinking, samples were swelled overnight in DPBS before mechanical testing. Compression tests were performed at room temperature using a TA Electroforce 5500 mechanical loading device (TA Instruments, USA) fitted with a $250 \mathrm{~g}$ load cell. The cross-sectional area of the sample was measured by imaging with a steromicroscope (LEICA MZ6) before the test. The sample was compressed between two stainless steel platens in an unconfined setting, and kept hydrated with a drop of PBS solution. The displacement of the upper plate was controlled by a ramp function, at a rate of $0.01 \mathrm{~mm} \mathrm{~s}^{-1}$, until a total displacement of $25 \%$ of the sample height was reached. The point of inflection of the loading curve was used to measure the sample height. ${ }^{17}$ Load and displacement measurements were converted into stress $(\sigma)$ and strain $(\varepsilon)$ data using the crosssectional area and height. The compressive modulus was then computed using the slope of the stress-strain curve between a strain of 0.1 and 0.15 strain, according to literature protocols. ${ }^{17}$ 


\subsection{Extrusion-based 3D printing}

Extrusion printing was performed on an Inkredible 3D bioprinter (CELLINK AB, Sweden) running custom g-code and using a tapered nozzle with inner diameter $0.20 \mathrm{~mm}$ (Nordsen EFD). Extrusion pressure and write speed were optimized at $125 \mathrm{kPa}$ and $300 \mathrm{~mm} / \mathrm{min}$ respectively and the layer height was $0.2 \mathrm{~mm}$.

\subsection{Sterility testing: Bacterial challenge}

An E. coli strain (Stb13) transformed with a plasmid containing the gene for resistance to ampicillin (VectorBuilder, Shenandoah, TX 77384, USA ) was cultured overnight (O/N) in Lennox Luria Broth (LB, Sigma Aldrich) with ampicillin (100 $\mu \mathrm{g} / \mathrm{ml}$, Sigma- Aldrich). The infection of materials was carried out by dropping $100 \mu \mathrm{l}$ of $\mathrm{O} / \mathrm{N}$ culture in $10 \mathrm{ml} \mathrm{LB}$ with an O.D. 1.0 at $600 \mathrm{~nm}$ onto $0.1 \mathrm{~g}$ of freeze-dried hydrogels inside a $50 \mathrm{ml}$ sterile tube (Corning). Considering an $\mathrm{OD}_{600}$ of 1.0 corresponds to $\sim 8 \times 10^{8}$ cells $/ \mathrm{ml}$, each hydrogel was infected with approx. $8 \times 10^{7}$ bacteria. Four materials were analysed: Gelatin, GelMA, HA, HAMA under four conditions: (i) non-infected, non-EtO-treated; (ii) non-infected, EtO-treated; (iii) infected nonEtO-treated; (iv) infected, EtO-treated. The non-infected groups were treated with $100 \mu \mathrm{l}$ of $\mathrm{LB}+$ ampicillin. At the end of the EtO cycle all the groups were resuspended in $20 \mathrm{ml} \mathrm{LB}+$ ampicillin. Bacterial growth was carried out for a subsequent $24 \mathrm{~h}$ at $37^{\circ} \mathrm{C}$. $100 \mu \mathrm{l}$ of a 1:10 dilution in LB + ampicillin of each sample was transferred to a 96 well plate. O.D. 600nm was read every hour for 6 hours on a CLARIOstar plate reader (BMG Labtech, Ortenberg, Germany). Pure LB + ampicillin was used as the reference.

\subsection{Cell viability}

2D assay: adipose derived human adipose-derived mesenchymal stem cells (hADSCs) were isolated and cultured as previously described, ${ }^{35}$ seeded into multiwell plates at a density of $4000 / \mathrm{cm}^{2}$ and cultured for $24 \mathrm{~h}$ in proliferation medium. The medium was then removed and replaced by medium containing 10\% w/v of either untreated GelMA or EtO-treated GelMA. The GelMA used for this experiment was EtO-treated one day before being exposed to the cells, representing the situation of high risk for residual EtO content. The hADSCs were cultured for a further $24 \mathrm{~h}$ before being assessed for metabolic activity using a CellTiter Blue (Promega, Wisconsin, United States) assay, following manufacturer's instructions with a $4 \mathrm{~h}$ incubation time at $37^{\circ} \mathrm{C}$. CellTiter-Blue solution was frozen at $-80^{\circ} \mathrm{C}$ until required. For the quantitative measurement, vials of the resultant CellTiter-Blue solution were thawed and 100 
$\mu 1$ duplicates were placed into a 96-well PE OptiPlate and read using 550-15/600-20 emission/excitation fluorescence wavelengths in a CLARIOstar plate reader (BMG Labtech, Ortenberg, Germany). Cell numbers were calculated based upon a calibration curve of known cell densities

3D assay: hADSCs were encapsulated in 10\% GelMA (EtO-treated, autoclaved or filtered) containing $0.1 \% \mathrm{w} / \mathrm{v}$ lithium-acylphosphinate (Tokyo Chemical Industry Co.) and photocrosslinked with $10 \mathrm{~s}$ exposure of $365 \mathrm{~nm}$ light at $700 \mathrm{~mW} / \mathrm{cm}^{2}$ as previously described. ${ }^{35}$ The cells were incubated for $7 \mathrm{~d}$ in proliferation medium. Metabolic activity was assessed through a CellTiter-Blue assay at days 1,3 , and 7 . The samples were incubated for $4 \mathrm{~h}$ at $37^{\circ} \mathrm{C}$ before the media sample was removed. $500 \mu \mathrm{L}$ of dimethyl sulfoxide (DMSO) (MP Biomedicals, California, United States) was added to the scaffolds and incubated for a further $1 \mathrm{~h}$ to retrieve the metabolized resorufin trapped in the scaffold. After the DMSO step, the CellTiter-Blue solution was frozen at $-80^{\circ} \mathrm{C}$ until reading. For the quantitative measurement, vials of the resultant CellTiter-Blue solution were thawed and $100 \mu \mathrm{L}$ duplicates were placed into a 96well PE OptiPlate and read using 550-15/600-20 emission/excitation fluorescence wavelengths in a CLARIOstar plate reader (BMG Labtech, Ortenberg, Germany).

\subsection{Chondrogenesis}

hADSCs were sourced from the infrapatellar fat pad of human donors as previously described. ${ }^{35}$ Chondrogenesis of hADSCs embedded in $5 \%$ w/v gelatin, $5 \%$ w/v GelMA and $0.5 \% \mathrm{w} / \mathrm{v} \mathrm{HA}+\mathrm{LAP} 0.1 \%$ and photo-cured at $365 \mathrm{~nm}$ at $700 \mathrm{~mW} / \mathrm{cm} 2$ for $10 \mathrm{~s}$ with a cell density of $5 \times 10^{6}$ cells $/ \mathrm{ml}$, was induced by culturing them in a chondrogenic medium consisting of high-glucose DMEM (Lonza), $100 \mathrm{Uml}^{-1}$ penicillin and $100 \mu \mathrm{g} \cdot \mathrm{ml}^{-1}$ of streptomycin (Gibco), Glutamax (Gibco), and 15 mM HEPES (Gibco), 1\% insulin-transferring-selenium (Sigma- Aldrich), $100 \mathrm{nM}$ dexamethasone (Sigma-Aldrich), $50 \mathrm{mgml}^{-1}$ ascorbate-2-phosphate (Sigma-Aldrich), $10 \mathrm{ngml}^{-1}$ TGF 33 (Peprotech), and $10 \mathrm{ng} \mathrm{ml}^{-1}$ BMP6 (R\&DSystems) as previously described. ${ }^{35}$ Media was changed twice weekly. The chondrogenic medium was freshly prepared before changing the media.

\subsection{Quantification of glycosaminoglycan $(G A G)$ and DNA}

To quantify GAG content, $0.5 \mathrm{~mL}$ Papain Extraction Reagent was added to each sample and incubated at $60^{\circ} \mathrm{C}$ for 3 hours with vortexing every hour, followed by centrifuge at $1 \times 10^{5} \mathrm{G}$ 
for 10 mins at room temperature. Supernatant was collected and $100 \mu \mathrm{L}$ of which was further mixed with $500 \mu \mathrm{L}$ DMMB reagent by vortex. $100 \mu \mathrm{L}$ of the mixture was added into 96 -well plate and the absorbance were read by ClarioStar microplate reader. Based on a calibrated standard curve of the standard solution of chondroitin-sulfate, the content of GAG was then determined by the ratio of absorbance read at $525 \mathrm{~nm}$ and $595 \mathrm{~nm}$.

DNA content was measured by using the same digested extract with Quant-iT PicoGreen dsDNA Reagents Kit. A standard curve was prepared with Lambda DNA in the Kit. $100 \mu \mathrm{L}$ of Papain extraction was mixed with $100 \mu \mathrm{L}$ PicoGreen, and $100 \mu \mathrm{L}$ of the mixture was added into ultravision 96-well plate. After incubating for 5 minutes at room temperature, the fluorescence was read using a plate reader set at PicoGreen with Ex 483-15 nm and Em 530$30 \mathrm{~nm}$ at 4.6 focal plate height, Gain 1500 .

\subsection{RNA extraction, Reverse transcription and qPCR analysis}

Total RNA isolation and RT-qPCR analysis was performed as previous reported (Onofrillo et al., 2018). Briefly, total RNA of hMSCs were extracted using TriZOL (Thermo Scientific) reagent and Direct-Zol RNA extraction Kit (Zymo Research),Following the manufacturer's protocols. Reverse transcription (RT) of $500 \mathrm{ng}$ of RNA for each sample was performed using High Capacity Reverse Transcription Kit (Thermo Scientific). The relative amounts of COL2A1, COL1A2, SOX9, and GAPDH RNAs were evaluated with TaqMan gene expression assay (Applied Biosystems, Foster City, CA, USA). RT-qPCR was performed on a QuantStudio6 Flex Real-Time PCR System (ThermoFisher Scientific) and relative quantification was calculated with the $2 \mathrm{E}^{-\Delta \Delta \mathrm{CT}}$ method.

\subsection{Confocal Microscopy}

Immunostaining was performed on the whole scaffold as previously described. ${ }^{24,35}$ Briefly, cell-laden samples were fixed using $4 \%$ paraformaldehyde for $30 \mathrm{~min}$ at room temperature. After being washed three times in PBS and permeabilized in PBT (0.1\% Triton in PBS) for 60 min,antigen retrieval was performed by adding $1 \mathrm{mg} / \mathrm{mL}$ hyaluronidase diluted in PBS and incubating $30 \mathrm{~min}$ at room temperature. After three washes of 5 min each in PBS, samples were dropped into blocking solution (10\% goat serum diluted in PBT) for $60 \mathrm{~min}$ at room temperature and then incubated overnight at $4{ }^{\circ} \mathrm{C}$ with mouse anti-human collagen II, diluted 1:250 in blocking solution. The next day, samples were washed five times for $10 \mathrm{~min}$ each and secondary antibody (anti-mouse IgG Alexa Fluor-647) diluted 1:100 in blocking solution was added and incubated for $2 \mathrm{~h}$ at room temperature. After five washes (5 mins each in PBS), 
samples were incubated with active labeling solution (Phall-FITC) 1:500 diluted in PBT and incubate $2 \mathrm{~h}$ at room temperature. After five washes (5 mins each in PBS), actin was labelled with Texas Red-X Phalloidin 1:100 diluted in PBS for $60 \mathrm{~min}$ at room temperature and nuclei were stained by incubation with $5 \mu \mathrm{g} / \mathrm{mL}$ DAPI diluted in PBSfor extra $60 \mathrm{~min}$ at room temperature. Samples were kept at $4{ }^{\circ} \mathrm{C}$ until confocal imaging was performed on a NikonA1R confocal microscope using a Nikon 20X Objective PLAN APO AirDIC NA 0.75 WD 1000 and z-intensity correction during the scan. Z-stacks of $2.5 \mathrm{um}$ each were processed for 3D rendering (volume view) using NIS-Elements software (Nikon, Amsterdam, Netherlands) and the Alpha-blending algorithm plug in.

\subsection{Statistical Analyses}

Statistical analyses were performed using Prism 7 (Graphpad Software Inc.). To test for statistical differences between multiple groups, one way ANalysis Of VAriance (ANOVA) was performed using Tukey's post-hoc test. Direct comparisons between two groups were performed using an unpaired t-test with Welch's correction. By convention statistical significance was considered to be reached for $\mathrm{p}<0.05$.

\section{Results and Discussion}

\subsection{Initial screening of sterilisation methods}

In preliminary work, we explored a range of sterilisation techniques for methacrylated hydrogels. Ethanol sterilisation was discarded because it did not result in a sterile material. Gamma irradiation was discarded as it resulted in noticeable colour change of the methacrylated materials (see supplementary information). Filtration was only practicable for GelMA, and not for gelatin, HA, or HAMA due to clogging of the 0.22um syringe filters. After this screening step, we chose to proceed with further examination of autoclaving and EtO treatments for all four materials, as well as filtration for GelMA only.

Autoclaving uses moist heat at high pressure to destroy microorganisms by the irreversible coagulation and denaturation of enzymes and structural proteins. However, besides eradicating microbes, the high pressure and temperature can severely degrade the materials being sterilised. The filtration method of sterilisation excludes organisms from a material based upon size. Filtration through a membrane with a pore size of $0.2 \mu \mathrm{m}$ will remove biological contaminants, 
including bacteria, mould and yeast, with sterile $0.22 \mu \mathrm{m}$ filters being the industry standard. ${ }^{36}$ Nevertheless some species of bacteria have been shown to be flexible enough to pass through $0.22 \mu \mathrm{m}$ filters. ${ }^{37}$

At room temperature and pressure, ethyelene oxide is a highly reactive gas which is lethal to mycobacteria, vegetative bacteria (gram-negative and gram-positive), bacterial spores, fungal spores, enveloped and naked viruses and prions. ${ }^{38}$ The mechanism of sterilisation is alkylation of sulfhydryl, amino, carboxyl, and hydroxyl groups, which damages proteins and nucleic acids. ${ }^{39}$ Inactivation of bacterial endotoxin by EtO has also been reported. ${ }^{40}$

\subsection{Effect of sterilisation methods on the rheology of bio-ink materials.}

Following the screening step, the chosen sterilisation methods were assessed for their impact on rheological properties of the four bio-ink materials. Formulating a printable bio-ink requires careful consideration of many rheological properties including viscosity, ${ }^{16,41}$ shear-thinning behavior, ${ }^{16,42}$ yield stress, ${ }^{25,42}$ viscoelasticity, ${ }^{7}$ shear recovery ${ }^{42}$ etc. We chose to quantify the yield stress and the viscosity at a particular shear rate $\left(1 \mathrm{~s}^{-1}\right)$ as representative rheological properties with which to monitor relative changes caused by sterilisation. (The viscosity at zero shear-rate is also reported in the supporting information. Within each data set these $0 \mathrm{~s}^{-1}$ data exhibit very similar trends to the viscosity at $1 \mathrm{~s}^{-1}$ for all materials). Rheological properties were assessed for untreated materials, autoclaved materials and EtO-treated materials for all four hydrogels (gelatin at 10\% w/v, GelMA at $10 \% \mathrm{w} / \mathrm{v}, \mathrm{HA}$ at $4 \% \mathrm{w} / \mathrm{vand} \mathrm{HAMA}$ at $4 \% \mathrm{w} / \mathrm{v}$ ), as well as the additional filtered group for GelMA.

Figure 1 (a-c) shows the effects of sterilisation on the rheological properties of gelatin solutions. The shear-ramp data presented in Figure 1 (a) describes similar rheological behavior for the untreated and EtO-treated gelatin, with both materials exhibiting increasing viscosity at low shear rates, reaching a peak of $~ 5000$ Pa.s, at which point the yield stress was reached and shear-thinning behavior ensued. The viscosity of autoclaved gelatin was dramatically lower, generally < 10 Pa.s at all shear rates, and appears only as a baseline viscosity on the scale of the plot. The quantitative analysis presented in Figure 1 (b-c) supports these two clear effects: (i) EtO treatment did not result in measurable changes in either yield stress or viscosity, with both properties showing no significant differences relative to the untreated materials. (ii) Autoclaving caused a dramatic reduction in both yield stress and viscosity, with both properties showing significant differences $(\mathrm{p}<0.01$ and $\mathrm{p}<0.0001$ respectively) relative to the untreated group. This effect of autoclaving is unsurprising since gelatin is known to degrade at elevated temperatures though hydrolysis of peptide bonds and internal cross-links. ${ }^{43}$ 
Figure 1 (d-f) shows the effects of sterilisation on the rheological properties of GelMA solutions. For autoclaving and EtO treatment, the results correlate with the effects obtained for the analysis of gelatin. Again, EtO treatment resulted in a similar shear-ramp profile to the untreated group, with similar (if slightly increased) yield stress values and a similar viscosity. Once again, autoclaving produced a dramatic reduction in both properties.

Gelatin 10\%: Rheology
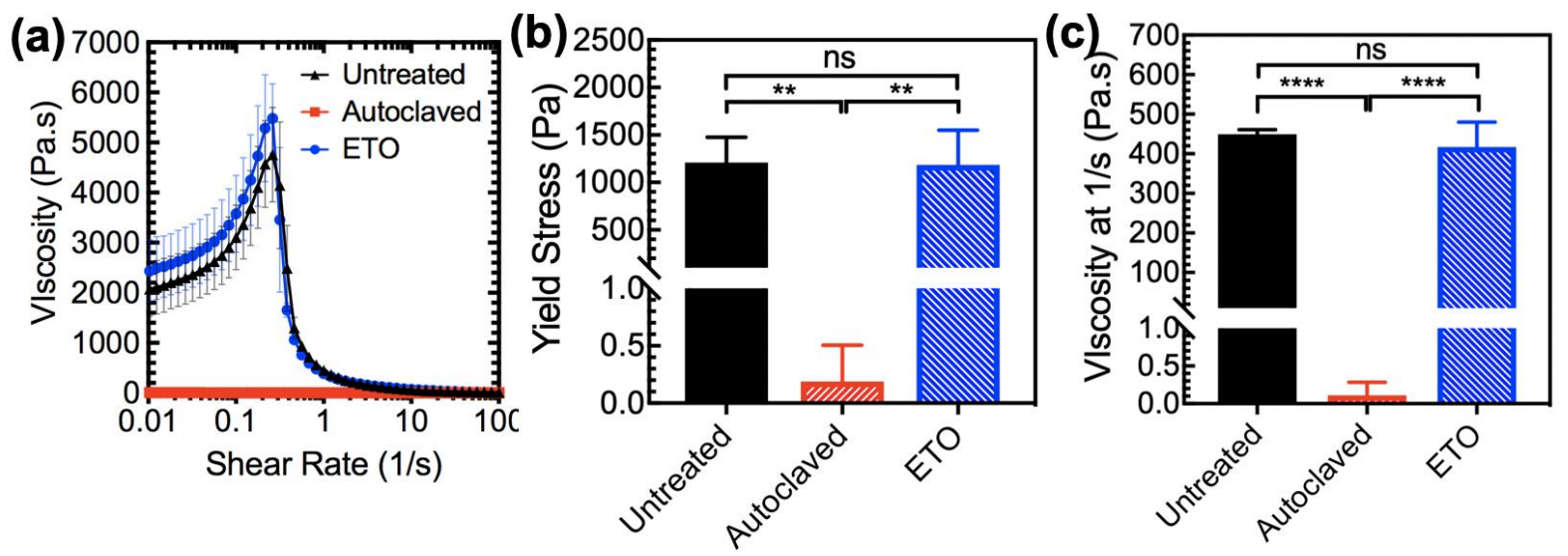

GelMA 10\%: Rheology

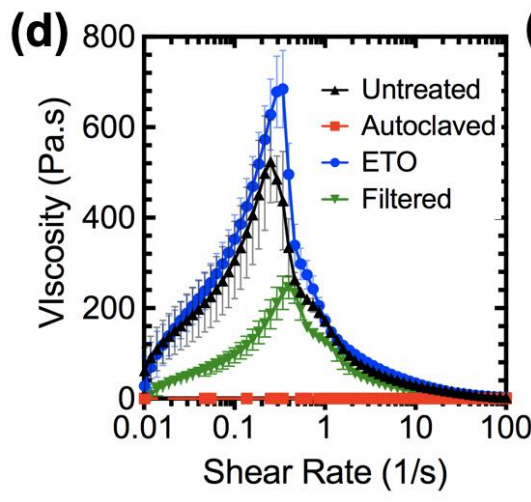

(e)
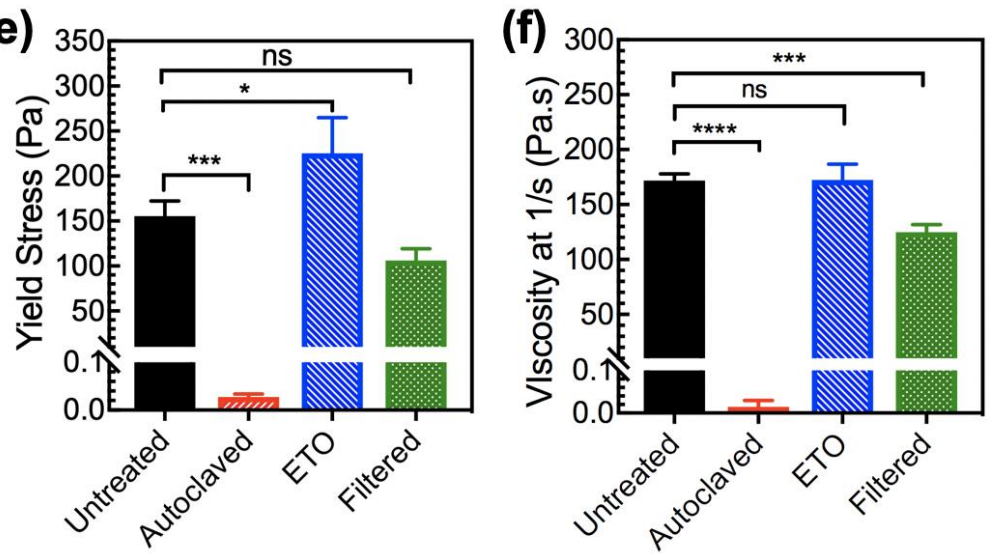

Figure 1: Effect of sterilisation on the rheological properties of gelatin 10\% w/v and GelMA $10 \%$ w/v solutions.

(a) Viscosity as a function of shear rate for gelatin materials. Both untreated and EtO-treated gelatin solutions showed increasing viscosity up to a peak of 4000-5000 Pa.s, with shear-thinning behaviour at higher shear-rates. The autoclaved gelatin, by contrast, had an extremely low viscosity (<10 Pa.s) at all shear-rates. (b) Yield stress for gelatin materials. Untreated and EtO-treated gelatin solutions exhibited a yield stress greater than $1000 \mathrm{~Pa}$, with no significant difference between them. Autoclaved gelatin showed a dramatically lower yield stress of below $0.5 \mathrm{~Pa}$. (c) Viscosity at a shear-rate of $1 / \mathrm{s}$ for gelatin solutions. Untreated and EtO-treated gelatin solutions had similar viscosities of $449 \pm 15$ Pa.s and $417 \pm 63$ Pa.s repectively, with no statistical significance between them. The viscosity of autoclaved gelatin was dramatically lower, at $0.11 \pm 0.17$ Pa.s. (d) Viscosity as a function of shear rate for GelMA materials. The viscosities of GelMA solutions were about an order of magnitude lower than for the gelatin solutions. Both untreated and EtO-treated GelMA solutions showed an increasing viscosity up to a peak of 500-700 Pa.s, with shear-thinning behaviour at increasing shear-rates thereafter. Autoclaved GelMA 
exhibited an extremely low viscosity (<1 Pa.s) at all shear-rates. (e) Yield stress for GelMA materials. Untreated GelMA solutions exhibited a yield stress of $155 \pm 17 \mathrm{~Pa}$ Pa, and EtO-treated GelMA a yield stress of $225 \pm 39$ $P a$, with a small statistical significance between them ( $p=0.02)$. Filtered GelMA had a yield stress of $106 \pm 13$ $\mathrm{Pa}$, with no significant difference w.r.t. the untreated material. Autoclaved GelMA showed a very low yield stress of just $0.027 \pm 0.006 \mathrm{~Pa}$. (f) Viscosity at a shear-rate of $1 / \mathrm{s}$ for GelMA solutions. Untreated and EtO-treated GelMA solutions had viscosities of $172 \pm 6$ Pa.s and $172 \pm 14$ Pa.s respectively, with no statistical significance between them. The viscosity of filtered GelMA was $125 \pm 7$ Pa.s, which was significantly different compared to both the untreated $(p=0.0007)$ and EtO treated $(p=0.0006)$ materials. The viscosity of autoclaved GelMA was dramatically lower than all other groups, at less than 0.03 Pa.s.

Notes: In graphs (a) and (d), black, upward-pointing triangles indicate untreated materials, red squares indicate autoclaved materials, blue circles indicate EtO-treated materials and green, downward pointing triangles indicate filtered materials. $n=3$ or greater for all groups. $* p<0.05, * * p<0.01, * * * p<0.001, * * * * p<0.0001$. Error bar represent standard deviation.

Interestingly, the rheological properties of the filtered GelMA group were reduced with respect to those of the untreated control. This could not be due to loss of mass during filtering, since all material was freeze-dried and weighed after the filtering step, before being re-dissolved at $10 \% \mathrm{w} / \mathrm{v}$. We hypothesized that the filtration was preferentially removing higher molecular weight molecules from the GelMA materials, which would reduce overall viscosity. To resolve the question, and to obtain an indication of the effects of the different sterilisation methods at the molecular scale, we performed gel permeation chromatography (GPC) and nuclear magnetic resonance (NMR) spectroscopyon the four GelMA samples (

\section{Table 1).}

NMR spectroscopy revealed that the degree of functionalisation was similar for all materials, indicating that the methacryloyl groups were not directly impacted by any of the sterilisation techniques. The NMR spectra were similar in all cases, indicating minimal chemical modification of GelMA by any treatment (see supporting information). From the GPC analysis, untreated GelMA was found to be composed of highly polydisperse macromolecules with a number average molecular weight $\left(\mathrm{M}_{\mathrm{n}}\right)$ of $\sim 41 \mathrm{kDa}$, a weight averaged molecular weight $\left(\mathrm{M}_{\mathrm{w}}\right)$ of $\sim 179 \mathrm{kDa}$ and a dispersity $\left(\mathrm{M}_{\mathrm{w}} / \mathrm{M}_{\mathrm{n}}\right)$ of 4.3 . For filtered GelMA, the $\mathrm{M}_{\mathrm{n}}$ stayed stable at 40 $\mathrm{kDa}$, while the $\mathrm{M}_{\mathrm{w}}$ decreased to $\sim 146 \mathrm{kDa}$. This indicates that smaller macromolecules had survived the filtering process, while larger macromolecules had been removed (thus explaining the reduction in viscosity observed for filtered GelMA). Autoclaving caused significant shortening (i.e. a $\sim 50 \%$ reduction) of the macromolecules of GelMA, yielding $\mathrm{M}_{\mathrm{n}}$ of $\sim 23 \mathrm{kDa}$ and $\mathrm{M}_{\mathrm{w}}$ of $\sim 83.5 \mathrm{kDa}$. This autoclave-induced degradation of GelMA is to be expected since high temperatures cause gelatin to degrade through hydrolysis of peptide bonds and internal 
cross-links. ${ }^{43}$ EtO-treated GelMA had a $\mathrm{M}_{\mathrm{n}}$ of $\sim 32 \mathrm{kDa}$ and $\mathrm{M}_{\mathrm{w}}$ of $\sim 161 \mathrm{kDa}$, indicating some reduction of larger molecular weight components, with a concomitant increase in the number of small macro molecules. 3 , In a study of the reaction of ethylene oxide with gelatin, Gantar et al. found that the prevailing reaction was addition of $\mathrm{EtO}$ onto the free $-\mathrm{NH}^{2}$ groups on the amino acids with basic side chains at neutral $\mathrm{pH}$ (i.e. lysine, arginine, aspartine) only. ${ }^{44}$ Such a reaction would have a minimal effect on molecular weight (though in our GelMA materials, about $70 \%$ of these groups are functionalised with methacrylamide). Thus we suggest that the slight decrease in molecular weight found in EtO treated GelMA may be due to hydrolytic degradation splitting some of the GelMA chains. Others have observed hydrolytic degradation of gelatin incubated at $30^{\circ} \mathrm{C}$ for 24 hours, conditions not dissimilar from our EtO sterilisation conditions $\left(25^{\circ} \mathrm{C}\right.$ over 24 hours). Altogether, EtO treatment had the smallest effect on molecular weight of GelMA, followed by filtering, with autoclaving the most detrimental.

Table 1: Gel permeation chromatography and NMR analysis of GelMA materials.

\begin{tabular}{l|cccc} 
& Untreated & Filtered & Autoclaved & EtO \\
\hline $\mathbf{M}_{\mathbf{n}}$ & 41390 & 40655 & 23287 & 32431 \\
$\mathbf{M}_{\mathbf{w}}$ & 178896 & 146260 & 83521 & 161300 \\
Dispersity & 4.32 & 3.60 & 3.59 & 4.97 \\
\hline $\begin{array}{l}\text { Degree of } \\
\text { functionalisation }\end{array}$ & $69.6 \%$ & $69.5 \%$ & $69.5 \%$ & $69.6 \%$
\end{tabular}

Figure 2 (a-c) shows the effects of sterilisation on the rheological properties of HA solutions. EtO-treated HA showed similar rheological behavior to untreated HA under shear ramp conditions, but with a slightly lower viscosity at all shear rates. The viscosity of autoclaved HA was less than half that of either untreated or EtO-treated HA at low shear-rates. Due to strong shear thinning behavior, all three HA groups approached similar viscosities at very high shear rates $\left(10-100 \mathrm{~s}^{-1}\right)$. The yield stress value for EtO-treated HA $(\sim 15 \mathrm{~Pa})$ was slightly lower than that of untreated HA ( 18 Pa), but this difference was not statistically significant. However, the yield stress of autoclaved HA ( 7 Pa) was far lower than either group, and these differences were significant $(p>0.01$ relative to EtO-treated and $p<0.001$ relative to the autoclaved group). The viscosity at $1 \mathrm{~s}^{-1}$ of the untreated HA was higher than that of either the EtO or untreated groups, which were similar. However, EtO-treated HA had a significantly higher zero-shear rate viscosity than autoclaved HA (see supporting information). This disparity is explained by the strong shear-thinning behaviour of HA. At very low or zero shear 
rates, HA molecules are entangled in random orientations, producing viscosities which are related to the HA chain length (with untreated $>$ EtO treated $>$ autoclaved). However as shear rates approach $1 \mathrm{~s}^{-1}$, viscosity drops due to shear-induced alignment of the HA molecules, and in this situation the viscosity is not as strongly affected by chain length (so the viscosities of untreated, EtO treated and autoclaved $\mathrm{HA}$ are relatively similar).

Figure 2 (d-f) shows the effects of sterilisation on the rheological properties of HAMA solutions. HAMA, in general, exhibited a much lower viscosity than the HA. In the shear-ramp plot, the viscosity of untreated HAMA was the highest at all shear-rates, followed by EtO treatment and then autoclaving. HAMA did not exhibit yield point behavior, and so the yield stress values for all HAMA groups were very low, and similar for all groups ( 2 Pa). The viscosity at $1 \mathrm{~s}^{-1}$ of the untreated HAMA was the highest ( 20 Pa.s) followed by EtO treatment ( 12 Pa.s) and finally the autoclaved HAMA group ( 8 Pa.s). The differences in viscosity between all groups were highly significant $(\mathrm{p}<0.0001)$.
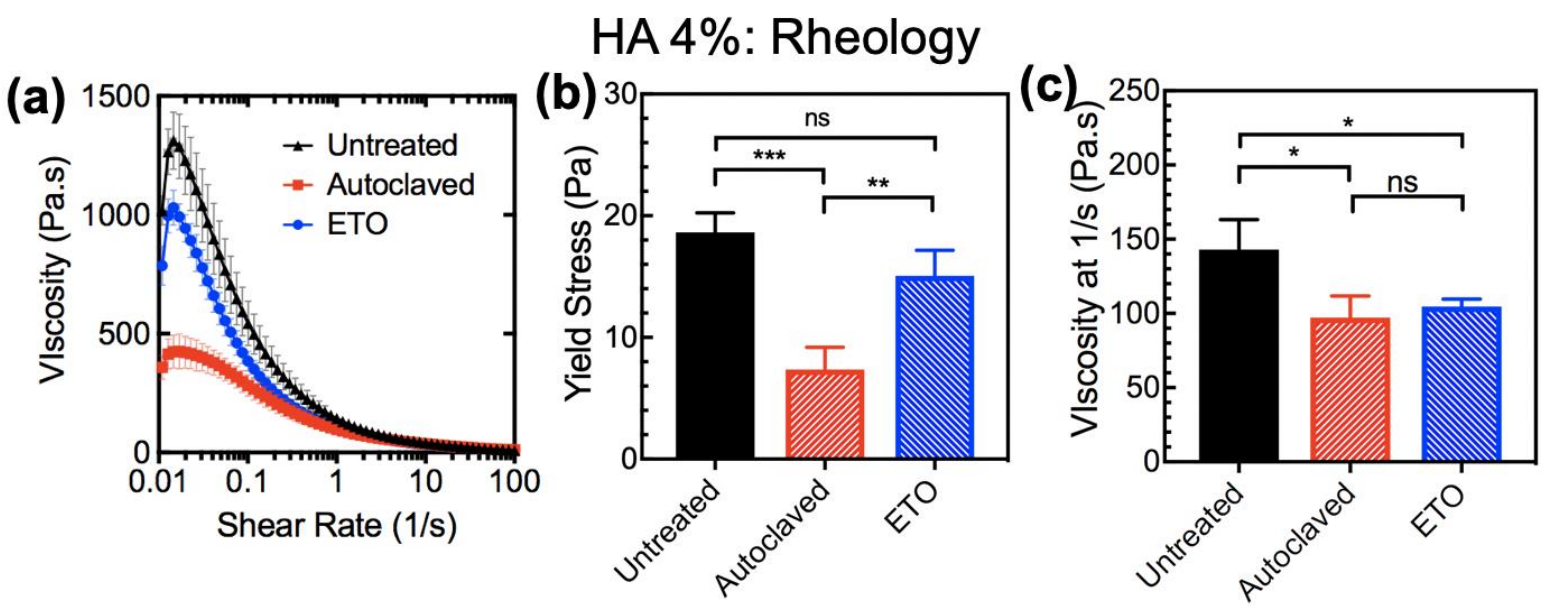

HAMA 4\%: Rheology
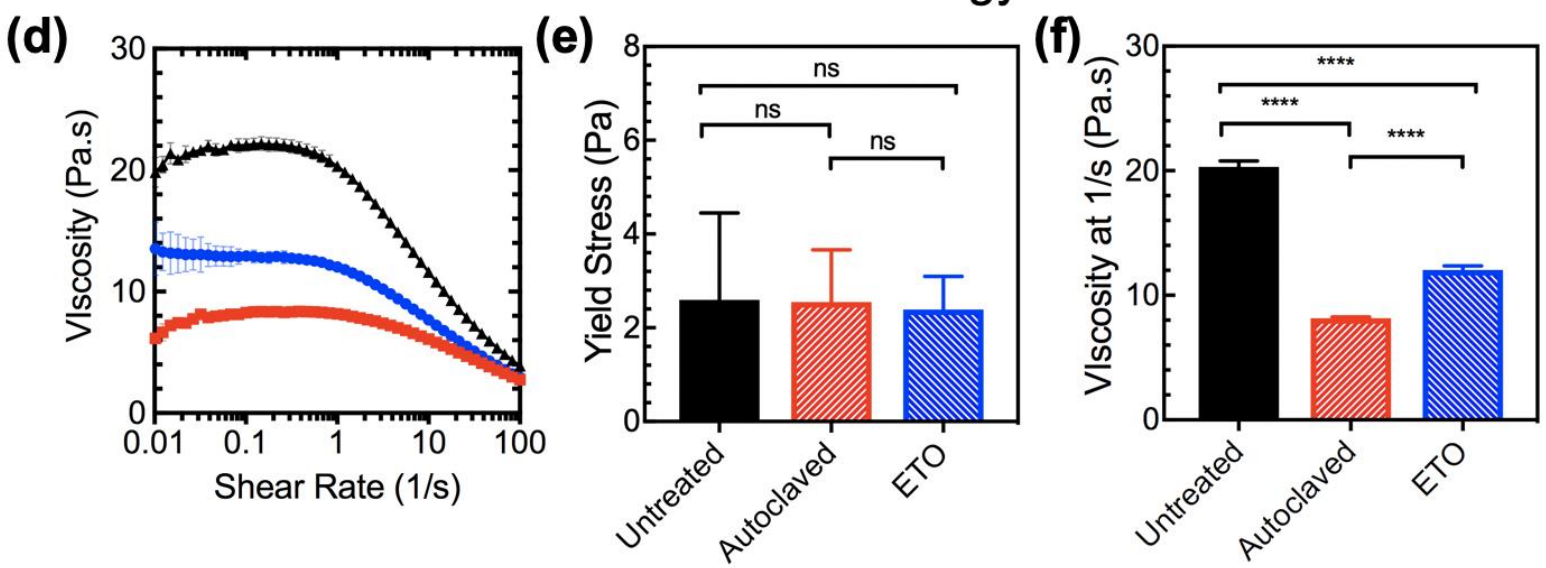

Figure 2: Effects of sterilisation on the rheological properties of HA $4 \% w / v$ and HAMA 4\% w/v solutions. (a) Viscosity as a function of shear rate for HA materials. Untreated and EtO-treated HA solutions showed increasing viscosities at low shear rates up to peaks of $1313 \pm 121$ Pa.s and $1030 \pm 73$ Pa.s respectively whereas autoclaved 
HA had a peak viscosity of just $425 \pm 73$ Pa.s, with shear-thinning behaviour at increasing shear-rates thereafter. (b) Yield stress for HA materials. Untreated HA solutions exhibited the highest yield stress (18.6 \pm 1.6 Pa), followed by EtO-treated HA (15.0 $\pm 2.1 \mathrm{~Pa})$, with no significant difference between them. Autoclaved HA had much lower yield stress $(7.4 \pm 1.4 \mathrm{~Pa})$, which is significantly different compared to both other groups $(p=0.0054$ vs EtO-treated and $p=0.0007$ vs untreated). (c) Viscosity at a shear-rate of $1 /$ s for HA solutions. Untreated HA exhibied the highest viscosity (143 \pm 20 Pa.s), which was significantly higher than the other groups. EtO-treated $(105 \pm 5 \mathrm{~Pa})$ and autoclave-treated HA $(97 \pm 15 \mathrm{~Pa})$ exhibited similar viscosities. (d) Viscosity as a function of shear rate for HAMA materials. The viscosities of HAMA solutions were dramatically lower than for HA. All materials showed a steady or slightly increasing viscosity at low shear rates, with shear thinning onset at a rate of $\sim 1 / s$. Untreated HAMA solutions were the most viscous, EtO-treated HAMA exhibited intermediate viscosity, and autoclaved HAMA had the lowest viscosity. (e) Yield stress for HAMA materials. HAMA solutions did not show yield point behaviour (as shown by the lack of a 'yield viscosity' peak in figure (d). All HAMA solutions thus exhibited a very low yield stresses of $2 \mathrm{~Pa}$, with no statistical signifance between any of the groups. (f) Viscosity at a shear-rate of 1/s for HAMA solutions. Untreated HAMA, EtO-treated HAMA and autoclaved HAMA had viscosities of $20.3 \pm 0.5$ Pa.s, $12 \pm 0.3$ Pa.s and $8.2 \pm 0.1$ Pa.s respectively, with significant differences $(p<0.0001)$ between all groups.

Notes: In graphs A and D, black, upward-pointing triangles indicate untreated materials, red squares indicate autoclaved materials, and blue circles indicate EtO-treated materials. $n=3$ or greater for all groups in all experiments. $* p<0.05, * * p<0.01, p<0.001$, ***** $p<0.0001$. Error bars represent standard deviation.

Our results show that autoclaving caused a large reduction in the rheological properties of the hyaluronic acid-based materials, a finding that agrees with literature reports. ${ }^{45,46}$ This reduction in viscosity is likely due to splitting of the HA molecule into shorter chains. HA is a large, rigid polysaccharide, but its molecular backbone can be quite easily depolymerised by splitting the (C-O-C) glycosidic bonds by heating, oxidation, and other effects. ${ }^{46}$ For example, Reháková et al. found the average molecular weight of HA decreased from $1.4 \times 10^{6}$ to $1.18 \times 10^{6}$ when heated to $90^{\circ} \mathrm{C}$ for one hour. ${ }^{46}$ Meanwhile Bothner et al., saw a reduction in both limiting viscosity number and molecular weight as a function of autoclaving time at $128^{\circ} \mathrm{C}$, with the kinetics of heat degradation in agreement with random scission of the HA chain. ${ }^{45}$ In contrast to the gelatin-based materials, the rheological properties of hyaluronic acid materials were also reduced by the EtO treatment. It is possible the glycosidic bonds are also susceptible to cleavage during EtO sterilisation, either due to the action of the EtO itself or by other species generated in reactions of EtO with water. While a detailed descriptiong of the mechanism of HA degradation is outside the scope of this study we note that, in neutral aqeous conditions, the epoxide ring opening reaction which converts EtO to ethylene glycol is achieved through hydrolysis with pathways for producing either $\mathrm{H}^{+}$and $\mathrm{OH}^{-}$species. ${ }^{47}$ Hyaluronan has been found to be particularly susceptible to cleavage by free radicals including both $\mathrm{H}^{+}$and $\mathrm{OH}^{-}{ }^{48}$ 
For example, Kvam et al. found that hydroxy radicals caused a ten-fold decrease in number averaged molecular weight of HA after one hour of exposure. ${ }^{49}$ Regardless, our results show that EtO treatement caused much less reduction in rheological properties of both HA and HAMA compared with autoclaving.

\subsection{Effect of sterilisation methods on the crosslinking of bio-ink materials.}

In this work, we investigated how various sterilisation methods affected the photocrosslinking of GelMA and HAMA as well as the enzymatic crosslinking of gelatin by transglutaminase. Figure 3 summarises these results. Neither filtration nor EtO treatment significantly affected the photocrosslinking of GelMA, with both groups reaching similar final storage modulus values as the untreated group. Autoclaved GelMA showed a significant decrease in mechanical properties, resulting in a storage modulus of about half that of the other groups. These results echo those seen in the rheological measurements of GelMA, where EtO and filtration both had a minimal effect but autoclaving caused a dramatic reduction in viscosity.

The final storage modulus achieved by HAMA solutions was significantly impacted by both EtO and autoclave treatment relative to the untreated group. While untreated HAMA reached a final storage modulus of $\sim 1500 \mathrm{~Pa}$, the EtO-treated group reached $\sim 1100 \mathrm{~Pa}$ and the autoclaved group reached only $\sim 600 \mathrm{~Pa}$. These results also echo those seen in the measurements of HAMA viscosity, where EtO caused some reduction in viscosity but not as much as autoclaving.

Importantly, these results suggest that the photocrosslinkable groups of methacrylate and methacrylamide are not being affected by the EtO treatment. In the case of GelMA, no change in photocrosslinking rate or final modulus was observed following EtO treatment. This is as expected considering EtO caused no reduction in the degree of functionalisation of GelMA and only a relatively small reduction in molecular weight. The reduction in the final storage modulus for EtO-treated HAMA are likely due to shortening of the HAMA chains, rather than deactivation of the photocrosslinkable groups. We have not found any published reports which discuss the effect of EtO on the photocrosslinking of methacrylated polymers. Several reports do mention EtO being used for sterilisation of methacrylated hydrogels, without specific investigation of its effect on the mechanical properties of the materials. ${ }^{21,32,50}$

Sterilisation by both EtO and autoclaving strategies caused small decreases in the mechanical properties of enzymatically crosslinked gelatin, however these differences were not significant. 
The compressive modulus of TG-crosslinked gelatin solutions was $\sim 28000 \mathrm{~Pa}$ for the untreated gelatin, $24000 \mathrm{~Pa}$ for the EtO-treated gelatin and $\sim 20000 \mathrm{~Pa}$ for the autoclaved gelatin. The minimal decrease in TGase crosslinking suggest neither the EtO or the autoclaving have a major impact on the structure to these groups. Transglutaminase crosslinks lysine and glutamine groups present on the gelatin. Previous work has reported that EtO reacts with lysine through hydroxyethylation of one or both of its primary amino groups, although these reactions are strongly dependent on the EtO exposure conditions and on the particular protein being assayed. ${ }^{51}$ Autoclaving has also been shown to reduce the concentration of both lysine and glutamine in casein proteins. ${ }^{52}$
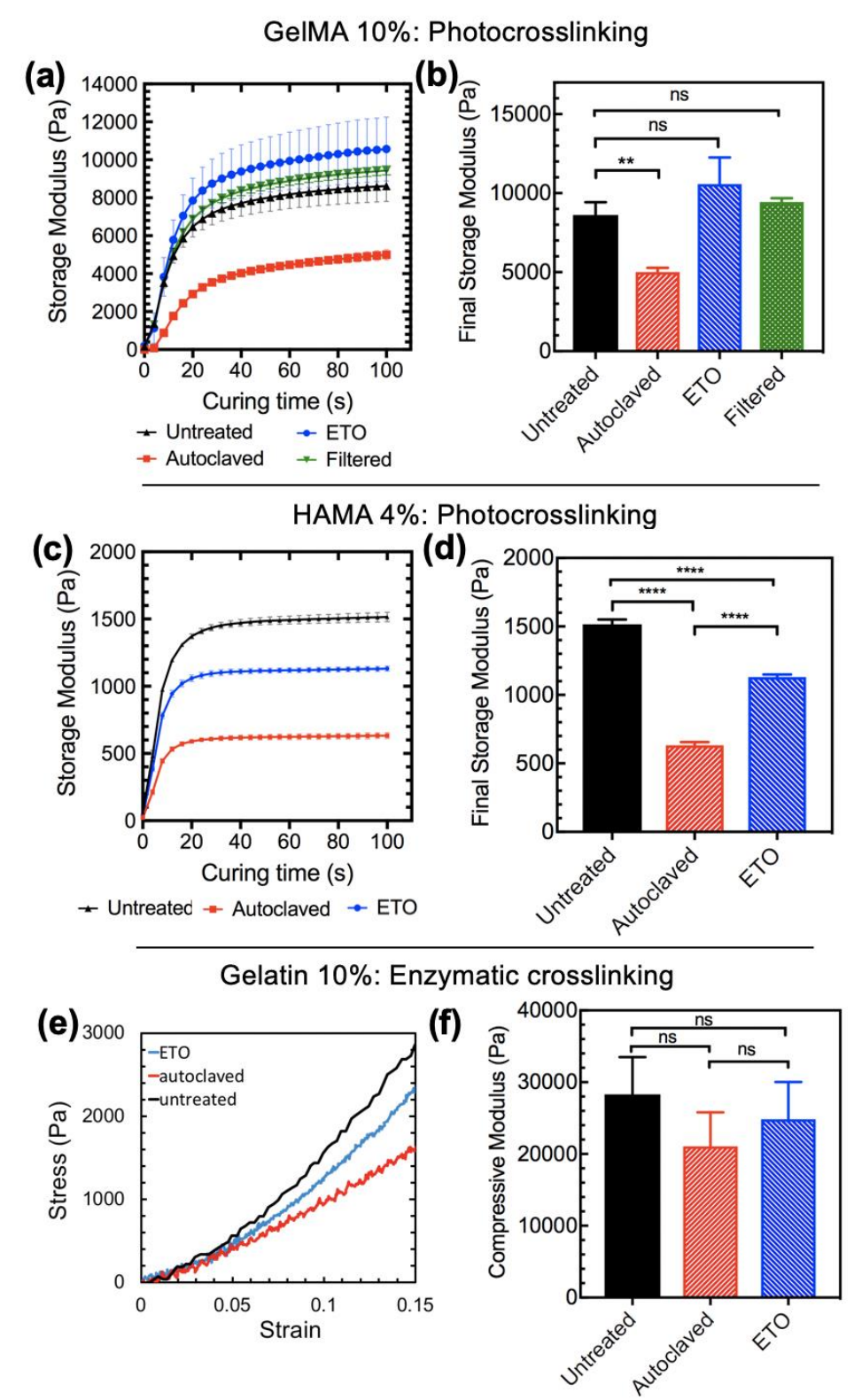

Figure 3: Effect of sterilisation on the crosslinking of hydrogels. (a) Photocrosslinking of GelMA $10 \%$ w/v solutions monitored using in-situ photo-rheology. Untreated, filtered and EtO-treated GelMA showed similar photo-crosslinking profiles. Autoclaved GelMA $10 \% \mathrm{w} / \mathrm{v}$ solutions showed a similar rate of reaction, but with a 
storage modulus less than half that of the other groups. (b) Final storage modulus values of cross-linked GelMA was in the range of 8000-1000 Pa for untreated, filtered and ETO-treated groups, with no statistical significance between any of them. However, the final storage modulus of autoclaved GelMA decreased markedly to 5000 Pa, a highly significant decrease compared to untreated GelMA ( $p=0.0007)$. (c) Photo-crosslinking of HAMA 4\% w/v solutions monitored using in-situ photo-rheology. There was a clear difference in photo-crosslinking profiles, with untreated HAMA producing the highest modulus, followed by EtO treatement and then autoclaving. The rates of reaction were similar, with all materials reaching plateaus after about 40 s of light exposure. (d) The final storage modulus of untreated, EtO-treated and autoclaved HAMA 4\% were $\sim 1500 \mathrm{~Pa}, \sim 1100 \mathrm{~Pa}$ and $\sim 600$ $\mathrm{Pa}$ respectively. There were highly significant differences between all three groups $(p<0.0001)$. (e) Typical stress-strain curves from compressive modulus measurements of gelatin $10 \%$ solutions crosslinked using transglutaminase. $(f)$ The compressive modulus of transglutaminase -crosslinked gelatin 10\% solutions was 28000 Pa for the untreated gelatin, 24000 Pa for the EtO-treated gelatin and 20000 Pa for the autoclaved gelatin, however there were no statistically significant differencse between any of these groups. Error bars represent standard deviation. $n=3$ or greater for all groups in all experiments.

\subsection{The election of EtO as an effective sterilisation method}

The results from all rheological and mechanical tests are summarized in Figure 4 below.

The most detrimental sterilisation strategy is autoclaving. Autoclaving caused a dramatic reduction in viscosity for all four materials. It also caused a large decrease in storage modulus for the photocrosslinked GelMA and HAMA, as well as a small (though non-significant) decrease in the compressive modulus of enzymatically crosslinked gelatin.

Filtering caused some reduction in rheological properties of GelMA, but did not affect photocrosslinking. Filtration also results in a significant loss of material: in our hands a $13 \%$ loss of material when manually filtered and a $~ 9 \%$ loss when filtered using a vacuum filtration system. Although filtration is the most common form of sterilisation reported for GelMA, challenges with scale-up, and its non-applicability to other, larger molecular weight materials are limitations.

Our results suggest EtO treatment is the most promising method for sterilizing all four bio-ink materials. EtO treatment seems especially suited for gelatin and GelMA, where it had minimal effect on the rheological properties and crosslinking for both of these materials. EtO is more detrimental to HA and HAMA, where it impacted negatively in terms of viscosity and (for HAMA) photocrosslinking. The decreases on these properties were still significantly less than those caused by autoclaving.

Besides these considerations of damage limitation during sterilisation, EtO treatment is also a highly scalable process. For materials which are not strong absorbers of EtO, the capacity of 
EtO sterilizers is limited only by the volume of the chamber. This means that relatively large quantities $(>1 \mathrm{~kg})$ of biomaterials can be sterilized in one cycle of a typical benchtop unit.

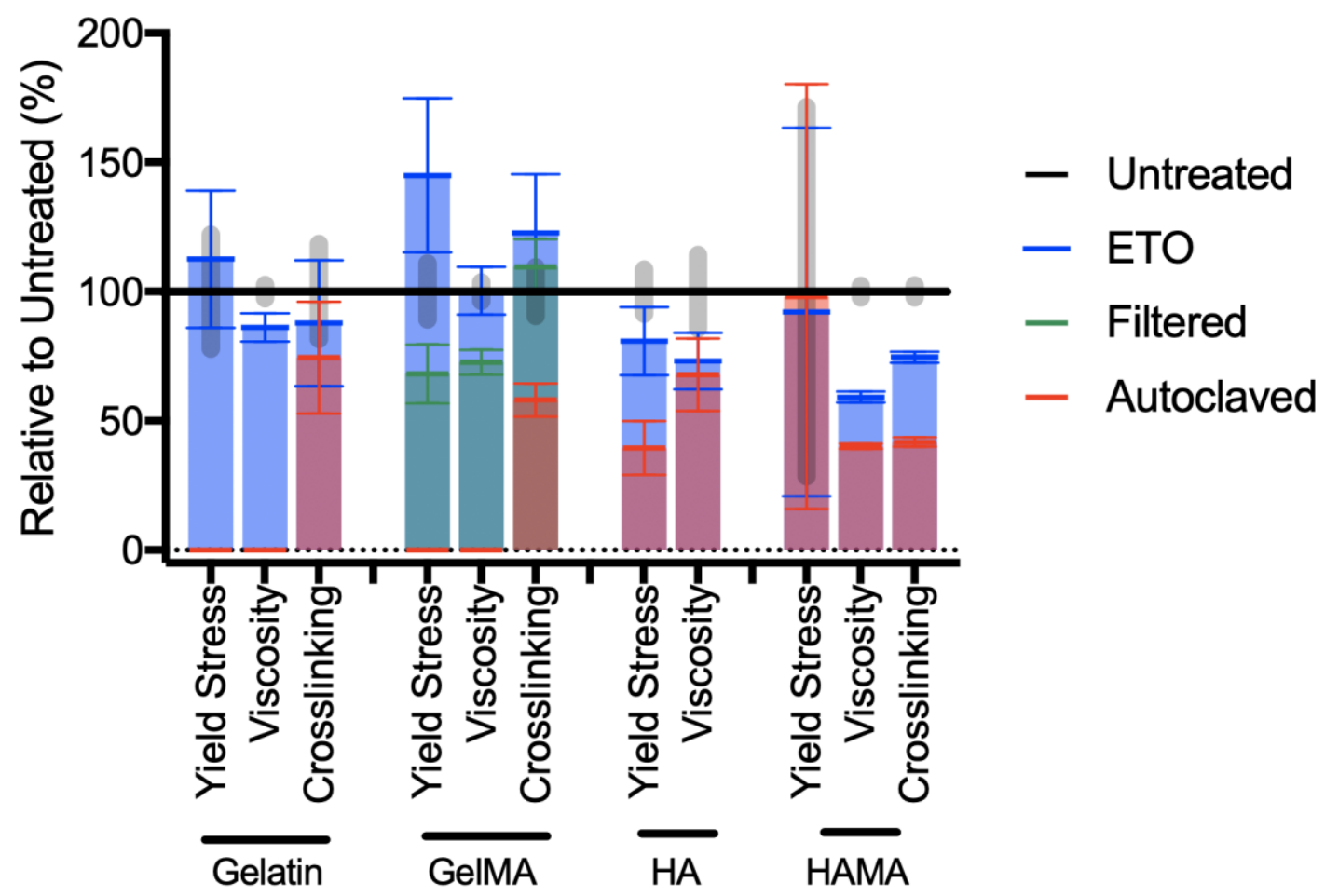

Figure 4: Summary of rheology and crosslinking measurements. All rheological and mechanical data are presented relative to each untreated group (normalised at $100 \%$, with error bars presented as grey ellipsoids). Gelatin and GelMA: In general, EtO treatment had little effect on the rheological and crosslinking properties of gelatin and GelMA materials. Filtration reduced the rheological properties of GelMA, without affecting crosslinking. Autoclaving dramatically reduced the rheological properies of both gelatin and GelMA, and also somewhat reduced crosslinking strength. HA and HAMA: EtO treatment reduced the rheological properies of HA and HAMA, although not as much as autoclaving. EtO treatment also reduced the final crosslinking modulus of HAMA, but again the impact of autoclaving was more detrimental. Error bars represent standard deviation.

\subsection{Bio-ink formulation}

Having characterised the properties of the four hydrogels individually, we next performed an experiment to illustrate how the choice of sterilision method can have a practical impact upon the bio-ink formulation process. With a view to developing a bio-ink for cartilage regeneration (as described in section 3.7 below) a base formulation of 5\% GelMA and $0.5 \% \mathrm{HA}$ was chosen. Various concentrations of gelatin (from $1-10 \% \mathrm{w} / \mathrm{v}$ ) were then incorporated, and the filamentforming ability of each formulation (a common screening test for printability $)^{5,9,53}$ was assessed at room temperature. (Both gelatin and HA are often used as bio-ink thickeners to increase viscosity and/or gelation, and so achieve printable bio-inks. $)^{5-9}$ 
We hypothesised that materials which retained higher rheological properties post-sterilisation (i.e. EtO sterilised materials) would achieve printability at lower additive concentrations. In this experiment we compared two groups of materials, one using materials which had been EtO-treated and the other using materials sterilised my other means. Our rationale here was to compare EtO treatment (of all materials) with the 'best case' or 'default' scenario chosen from all other sterilisation methods: namely filtration for GelMA, and autoclaving for gelatin and HA.

As shown in Figure 5 below, there is a stark difference in the printability between the two groups. While the EtO-treated bio-inks could form a filament when just $5 \%$ w/v gelatin was added, the filtered/autoclaved group did not form an extruded filament until 10\% w/v gelatin was added. Furthermore EtO-treated hydrogels generally produced much longer filaments than the filtered/autoclaved group, and at lower concentrations of gelatin. ${ }^{9}$ In particular, the filament length achieved by the filtered/autoclaved materials with $10 \%$ added gelatin was similar to that achieved by the EtO-treated materials with half that amount (5\%) of added gelatin. We also confirmed the printability of the optimised EtO treated formulation (5\% GelMA, 0.5\% HA, $5 \%$ gelatin) by 3D printing various structures including the $6 \mathrm{~mm}$ tall, 30-layered log-pile structure shown in Figure 5 (c-d). 


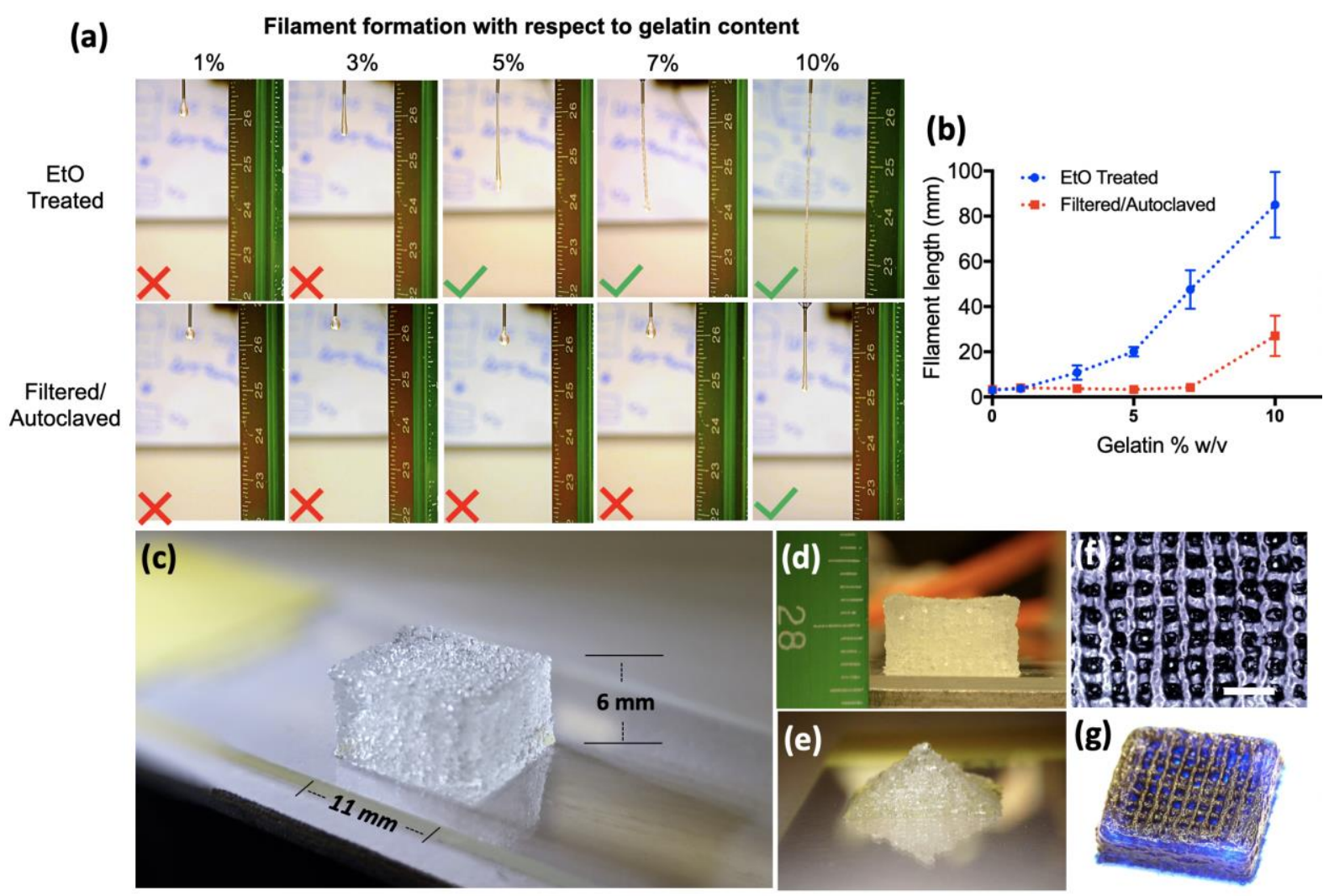

Figure 5: Bio-ink formulation using sterilised hydrogels. (a) Printability screening using the filament-formation test. For the EtO-treated group, filament formation was achieved when the base formuation (5\% w/v GelMA and $0.5 \% \mathrm{w} / \mathrm{vHA}$ ) was supplemented with gelatin to a final concentration $5 \% \mathrm{w} / \mathrm{v}$ gelatin. The filtered/autoclaved group required double this addition of gelatin $(10 \% \mathrm{w} / \mathrm{v})$ to form extruded filaments. (b) Measurement of the length of the extruded filaments at break-point, as a function of added gelatin. EtO-treated hydrogels generally produced much longer filaments than the filtered/autoclaved group, and at lower concentrations of added gelatin. Error bars represent standard deviation. A minimum of $n=3$ filaments were measured for each condition. (c) 30 layer, $6 \mathrm{~mm}$ tall log-pile structure printed using a bio-ink formulated from EtO treated materials (GelMA 5\%, gelatin 5\%, HA $0.5 \% \mathrm{w} / \mathrm{v}$ ). (d) Photograph of the log-pile from (c) next to a mm-scale ruler to demonstrate its 6 mm height.. (e) Stereomicroscope image of a 3D printed pyramid (22-layers) using the same formulation. (f) ) Lines in a crosshatch pattern printed using the same EtO treated bio-ink. The printed lines are $268+/-34 \mu m$ (measured from $n=10$ lines). (g) Stereomicroscope image of a $2.4 \mathrm{~mm}$ tall, 12 layer log-pile printed using the EtO-treated bio-ink and dyed blue after printing to better visualise this highly transparent structure. Note: All images in (c-g) were taken prior to any photocuring, showing the ability of the bio-ink to maintain shape fidelity without relying on chemical crosslinking.

Considering these results, the remainder of this study focuses on EtO treatment only, and on the biological characterisation of EtO-treated materials in particular. In our next step, we tested the capability of EtO as a sterilisation method for gelatin, GelMA, HA and HAMA, through issuing a bacterial challenge prior to treatment. We also assessed the viability proliferation and differentiation of human MSCs exposed to EtO-treated materials. 


\subsection{Contamination challenge for EtO-treated bio-inks}

To test whether EtO treatment could sterilise contaminated bio-ink materials, we deliberately infected samples of gelatin, GelMA, HA and HAMA with E. Coli prior to EtO treatment. Besides the infected and EtO-treated samples, three relevant control groups were assayed for each material, making for four experimental groups in total: (i) uninfected/untreated, (ii) uninfected/EtO-treated, (iii) infected/untreated, (iv) infected/EtO-treated.

Following $24 \mathrm{~h}$ incubation in LB, the relative abundance of bacteria in a 10 fold dilution of solution was assayed by optical density at $600 \mathrm{~nm}$. As shown in Figure 6 (a-d), the concentration of bacteria were far higher in the infected/untreated groups than for any other group for all four materials. To confirm that these bacteria were alive and proliferating, a bacterial growth curve was then measured from aliquots of each solution, diluted 10-fold in LB. As shown in Figure 6, the only group which permitted any bacterial growth was the infected/untreated group. In contrast, the OD for the infected/EtO group tracked with the OD for the uninfected controls. The strong contrast between the levels of bacterial growth can be seen in Figure 6 (i-1), which shows photographs of each LB solution 72 hours after the day of infection and treatment. While the infected/untreated solutions are densely clouded, the infected/EtO solutions are clear, similar to the uninfected and untreated controls. From these results, we conclude that EtO treatment effectively reduced the concentration of viable bacteria to below detectable levels for all four materials. 

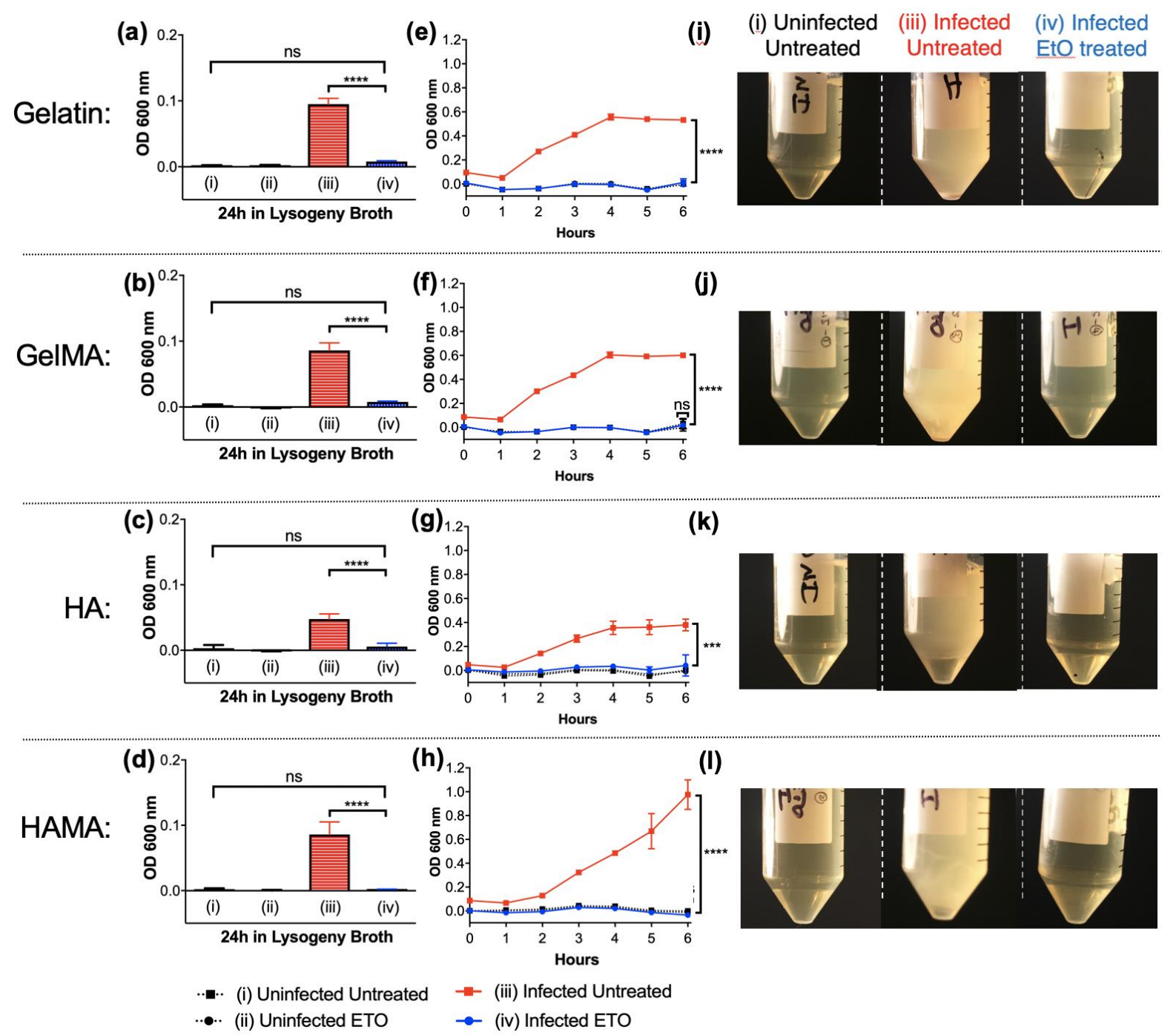

Figure 6: Sterilisation test for EtO treatment. Four conditions were tested for each material: (i) uninfected untreated, (ii) uninfected EtO-treated,(iii) infected untreated, (iv) infected-EtO-treated. (A-D) Initial bacterial growth in gelatin, GelMA, HA and HAMA respectively. Optical density reading at $600 \mathrm{~nm}$ following $24 \mathrm{~h}$ incubation in $L B$. The OD reading for the infected/untreated group wers far higher than any of the other groups for each material, and showed a statistically significant difference $(p<0.0001)$ relative to the infected/EtO group in each case. (E-H) Bacterial growth curves for gelatin, GelMA, HA and HAMA respectively. Following the initial $24 \mathrm{~h}$ incubation, aliquots of each solution were diluted in LB to monitor bacterial growth over time. For all materials, the only condition that showed an increased OD over time was the infected-untreated group. No bacterial growth was detected in the infected/EtO-treated samples for any of the materials. At the $6 \mathrm{~h}$ timepoint the differences between the the infected/untreated and the infected/EtO groups was highly significante ( $p<0.0001$ or $p<0.001)$. Notes: The data are corrected against the O.D. $600 \mathrm{~nm}$ of the culture broth at each timepoint. $n=3$ samples were taken for each condition, which were derived from the same infection event. For all conditions except HAMA bacterial growth reached stationary phase by 4 hs. Error bars represent standard deviation. (I-L) Images of infected/untreated, infected/EtO-treated and uninfected/untreated solutions $72 \mathrm{hs}$ after the infection and treatment. The cloudy appearance of the infected/untreated group indicates the abundance of bacteria. The uninfected/untreated and infected/EtO-treated groups, in contrast, are clear solutions. Note: The yellow colour of all solutions arises from $L B$. 


\subsection{Cell Viability within EtO-treated hydrogels}

Having established the efficacy of the sterilisation process, our next step was to look for any detrimental effect of EtO-treated hydrogels on the viability of human cells in culture. Questions are often raised about latent toxicity of EtO-sterilisation, because EtO is known to be absorbed by some materials, particularly plastics. Indeed, some polymers sterilised with EtO have been shown to retain high levels of the chemical even after 15 days of aeration. ${ }^{54}$ As a consequence of these concerns, standard protocols for EtO sterilised medical devices designate steps to ensure removal of residual EtO prior to use and set maximum allowable residue limits following sterilisation. ${ }^{55,56}$ The danger of residual EtO is likely mitigated in hydrogels since EtO reacts with water to produce ethylene glycol, which has negligible cytotoxicity at very low concentrations. ${ }^{57}$ Nevertheless, we must also consider the possibility that reactions involving EtO and hydrogels may produce other cytotoxic by-products.

In this work, we assessed whether the sterilisation process had any adverse effects on cell viability or proliferation, by performing both $2 \mathrm{D}$ and $3 \mathrm{D}$ cell culture studies using GelMA as a representative material.

Firstly, we used the 'direct contact' method to assess whether exposure to EtO-treated GelMA caused any immediate cell morbidity, as may be expected if residual EtO were present. ${ }^{58}$ The GelMA used for this experiment was EtO-treated one day before being exposed to the cells, representing high risk for residual EtO content. hADSCs were seeded in multiwell plates and cultured for $24 \mathrm{~h}$ in proliferation medium. The medium was then removed and replaced by medium containing 10\% w/v of either untreated GelMA or EtO treated GelMA. The hADSCs were cultured for a further $24 \mathrm{~h}$ before being assessed for metabolic activity using the CellTiter Blue assay. As shown in Figure 7 (A), the numbers of live cells in the untreated GelMA and EtO-GelMA were similar after this $24 \mathrm{~h}$ exposure, indicating that the EtO treatment had no measurable cytotoxic effect at this time point.

We then assessed the proliferation of cells encapsulated in photo-crosslinked GelMA in 3D, as would be used in the intended bioprinting application. This experiment compared metabolic activity of MSCs in filtered, autoclaved and EtO-treated GelMA over time in culture. As shown in Figure 7 (B), the increase in numbers of hMSCs encapsulated in all three GelMA groups did not show any significant differences in fold-changes by day 7 . We conclude that EtO treatment of GelMA had no significant detrimental impact on the survival of encapsulated hMSCs. 

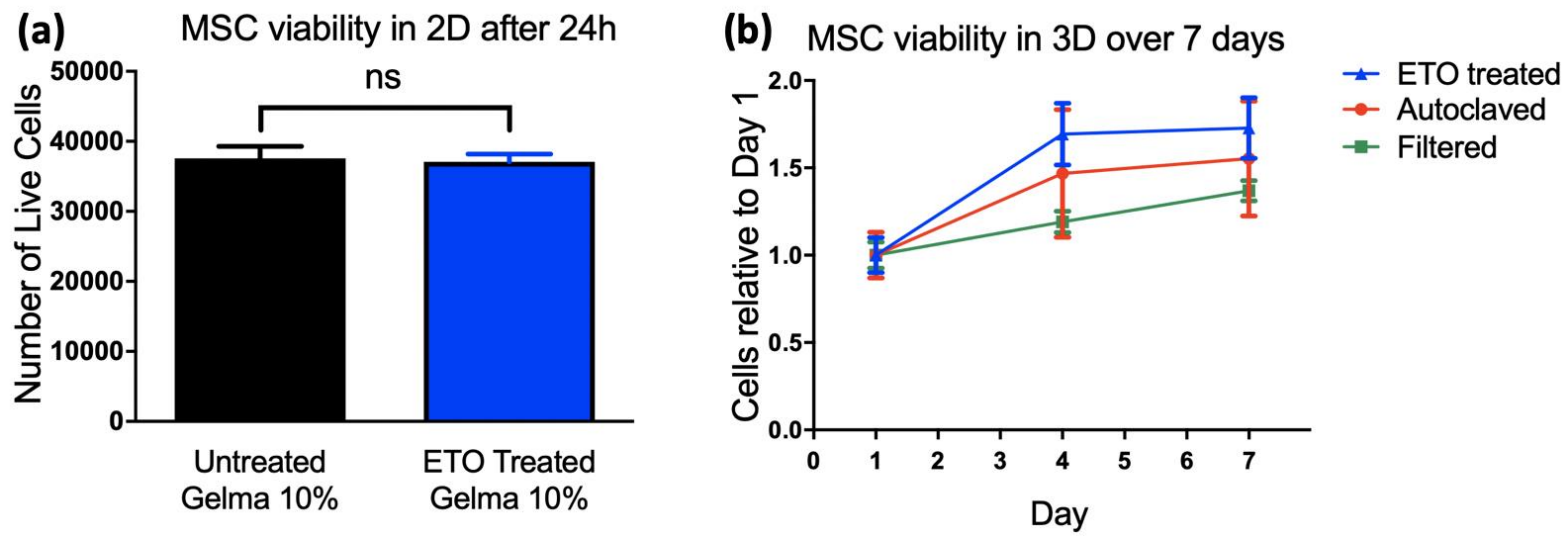

Figure 7: Viability of human mesenchymal stem cells in GelMA materials in 2D and 3D. (a) The viability of hMSCs in 2D culture was assessed by a metabolic assay (cell titer blue) after 24 h exposure to both untreated and EtO-treated GelMA at 10\% w/v in the culture medium. There was no statistical significance between cell numbers in the two groups $(p=0.65) . n=4$ for both groups. Cell numbers were calculated based upon a calibration curve of known cell densities. (b) hMSCs were encapsulated in photocrosslinked GelMA $10 \%$ w/v hydrogels (filtered, autoclaved and EtO-treated) and cultured for one week. Relative cell number was assessed at day 1, 4 and 7 by metabolic assay (cell titer blue). The increase in cell number was similar in all groups, with no statistically significant differences between relative cell numbers across any of the three groups by day $7(n=3)$.

These results agree with previous work which found no effect of EtO-treated HA on the viability of either mouse fibrobast (3T3) cells or human coronary venular endothelial cells. ${ }^{58}$ This was despite the detection of very low levels of EtO residues $\left(2.7 \times 10^{-2} \mu \mathrm{g} / \mathrm{ml}\right)$ after a 7day aeration period. Similarly, chitosan-membranes treated with EtO had no cytotoxic effect on Vero cells. ${ }^{59}$ In contrast to these studies, an EtO-treated copolymer of (50:50) poly-lacticpolyglycolic acid was determined to be cytotoxic to mouse L929 cells and human fibroblast cells, even after 14-days degassing under vacuum. ${ }^{60}$ Clearly, the levels of residual EtO are highly-dependent on the material being sterilised. In the case of hydrogels, our results and those previously reported suggest that residual EtO, if any, has no negative affect on cell viability.

\subsection{Biofabrication potential: Chondrogenesis of hMSCs within EtO-treated hydrogels}

Our final step was to evaluate the potential for EtO-sterilised hydrogels as materials for biofabrication and tissue engineering. We chose chondrogenic differentiation of human mesenchymal stem cells and neocartilage formation as an example application. Gelatin, ${ }^{61-63}$ GelMA, ${ }^{8,16,64-66}$ HAMA $^{16,64,67}$ and $\mathrm{HA}^{68-71}$ have all been explored as materials for cartilage engineering. Informed by the printability tests described in section 3.4 above, a biomaterial containing $5 \% \mathrm{w} / \mathrm{v}$ gelatin, $5 \% \mathrm{w} / \mathrm{v}$ GelMA and $0.5 \% \mathrm{w} / \mathrm{v}$ HA was formulated for the chondrogenesis study. All materials were EtO-treated. Owing to the fact that different 
sterilisation methods had varying impacts upon the mechanical properties of the hydrogels (section 2.6 above) and the challenge of delineating these effects on chondrogenesis of hMSCs, we did not perform a comparative study of EtO-treated and otherwise treated materials. This experiment is thus only an illustrative example, which showcases that EtO-treated hydrogels can be used in an in vitro tissue engineering context.

Based on previous work, we have observed that hADSCs encapsulated in hydrogels showed a genetic response to chondrogenic stimuli in vitro only after two weeks of culture, followed by increased expression of chondrogenic markers for up to 8 weeks. ${ }^{35,72}$ In this study we compared the standard markers for chondrogenesis (gene expression, GAG content and mechanical properties) on day 0 and day 26 of culture in chondrogenic medium.

As shown in Figure 8 below, the gene expression levels for key chondrogenic markers were upregulated during the 26 days of culture in EtO GelMA. On day0, no collagen II expression was detected, but it was clearly present on day26. In agreement with the increase in collagen II, there was a three-fold increase in the expression of its upstream regulator SOX-9 on day 26 relative to day 0 . Collagen II is a characteristic component of hyaline cartilage tissue, ${ }^{73}$ while SOX-9 is the transcription factor responsible for the activation of collagen II expression and the master regulator of chondrogenesis. ${ }^{74}$ The expression of collagen II and SOX-9 on day 26 is indicative of hyaline chondrogenesis as previously observed in hydrogels which had been filter sterilised. ${ }^{35}$

Glycosaminoglycans (GAG) are an important and characteristic component of cartilage tissue and play a critical role in cartilage mechanical properties. ${ }^{75}$ On day 0 and day 26 of chondrogenesis, scaffolds were digested to detect the content of glycosaminoglycan (GAG) and DNA. As shown on Figure 8 (d-f), the GAG content showed a 13-fold increase from day $0(1.20 \pm 0.10 \mathrm{ug})$ to day $26(16.0 \pm 1.96 \mathrm{ug})$. A 2.5 -fold increase in DNA amount was found when comparing samples from day 0 to day 26 , which suggests proliferation during the culture period. Since DNA amount often remains same for the same cell line, it can also be used as a reference for cell number. ${ }^{76}$ The GAG increase was statistically significant after normalization to the DNA amount. This result complies with our recent study using a similar approach with filter sterilised GelMA/HAMA and suggests neocartilage formation. ${ }^{35}$

Mechanical compression tests was also conducted on day0 and day26 of chondrogenesis. As shown in Figure $8(\mathrm{~g})$, below, there was an approximately 1.8-fold increase in compressive modulus from day $0(\sim 6 \mathrm{kPa})$ to day $26(\sim 11 \mathrm{kPa})$, and this increase was statistically significant. This result supports the finding from gene-expression and GAG analysis that the encapsulated cells are producing new extracellular matrix (ECM). 
Confocal imaging performed on day 26 is also shown. Nuclei (white) and actin (red) stainings were used to localize the position of cells and their polygonal morphology in the hydrogel scaffolds, while Collagen II (green) staining demonstrates the accumulation of cartilage ECM. Taken together, the results from RT-qPCR, GAG content, mechanical testing and confocal microscopy, suggest the seeded hMSC have entered a chondrogenic state within the EtO-treated hydrogels, consistent with other studies using filter sterilised materials. ${ }^{35,77}$
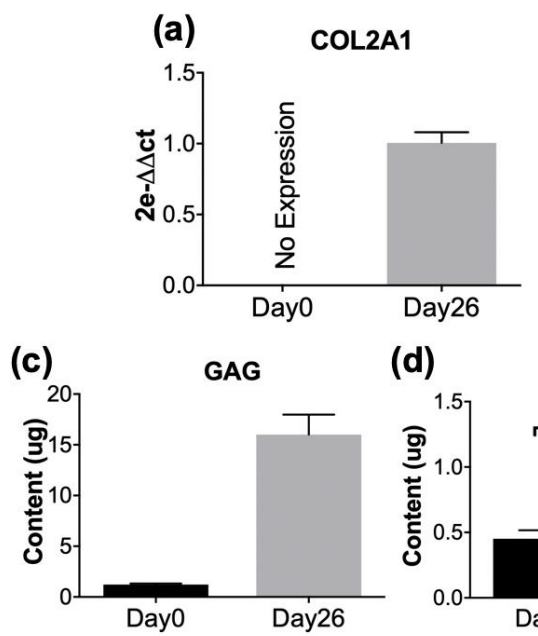

(b)

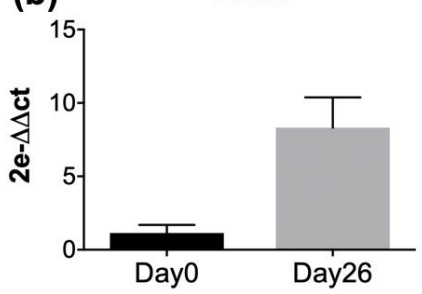

(d)

(e)

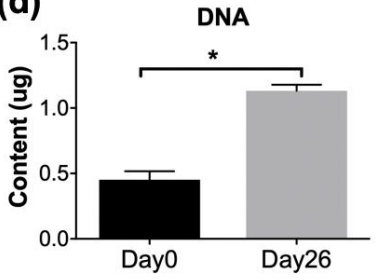

GAG/DNA
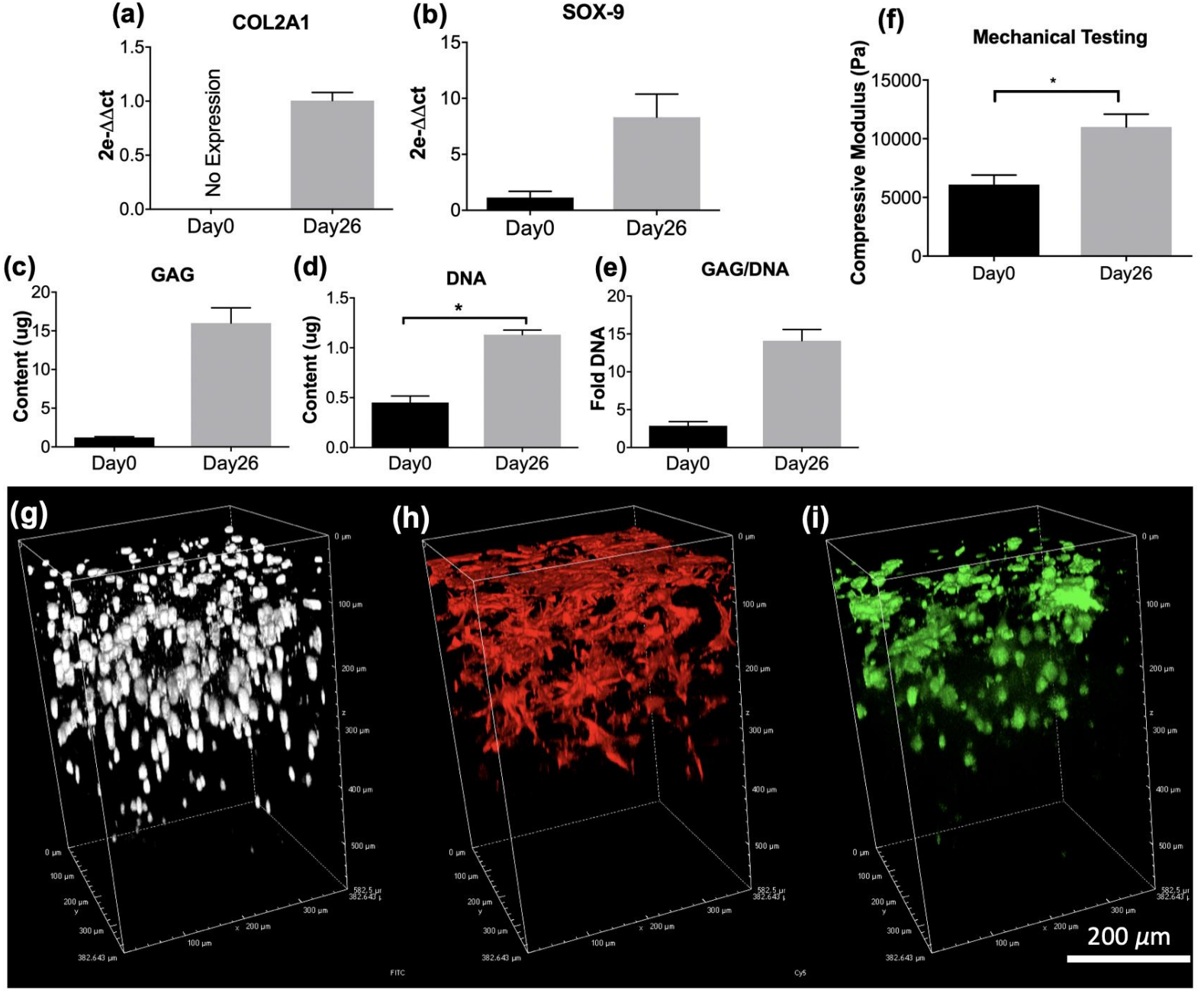

Figure 8: Utilisation of EtO-treated materials for a cartilage biofabrication strategy. (a-b) rt-qPCR analysis of expression of COL2A1 and SOX-9 before and after chondrogenesis. Results are presented as $2 \mathrm{e}-\Delta \Delta \mathrm{Ct}$ in order to present the fold change in expression at day 26 . Since no expression of COL2A1 was detected on day 0 , the fold change in COL2A1 could only be expressed as 1 (c-e) Result of fluorescence assay detecting: (c) glycosaminoglycan (GAG) content; (d) DNA content and; (e) the GAG/DNA, before and after chondrogenesis. Content was determined using a calibration curve. (f) Compressive modulus of hydrogels before and after chondrogenesis. *: p<0.05. (g-i) Confocal microscope images showing staining of: (g) cell nuclei; (h) actin filament; (i) collagen II. Axis directions arelabelled in G, where z represents the depth of sample. The scanning was performed from the top surface towards the bottom. Note: light attenuation from top to bottom precluded the clear analysis of material deeper than $\sim 300 \mu \mathrm{m}$. Notes: $\mathrm{n}=3$ for $(\mathrm{a}-\mathrm{f})$; $\mathrm{n}=1$ for $(\mathrm{g}-\mathrm{i})$ 


\section{Conclusions}

The field of biofabrication is entering a new phase of maturity with pioneering programs of pre-clinical and clinical trials under way. ${ }^{64,78-80}$ This phase is marked by new translational challenges, among them the requirements to demonstrate reliable and effective sterilisation of materials. In this work, we explored the effect of three sterilisation techniques on four typical bio-ink components. Our results suggest EtO treatment is the most promising method for sterilizing bio-ink hydrogels: with a much lower modulation of physical and chemical properties relative to autoclaving, and being more widely applicable than filter sterilisation. In a practical example targeting the bio-ink formulation process, we found EtO-sterilised bio-inks can achieve the standards for printability using far lower hydrogel concentrations than bio-inks sterilised by other means. Continuing with biological testing of EtO treatment only, we found that EtO could eradicate a dense bacterial contamination ( 80 million cells per $0.1 \mathrm{~g}$ of material) of all four hydrogels, that it caused no decrease in viability of human MSCs, and nor did it slow their rate of proliferation. Finally, we demonstrated that EtO-treated biomaterials supported hMSCs differentiation towards the chondrogenic lineage, and to produce new cartilage matrix.

In terms of physical, chemical and biological properties, ethylene oxide sterilisation appears to be a viable option for large-scale sterilisation of these naturally-derived bio-ink hydrogels. It also has a long history of use with generic implantable medical devices. ${ }^{39}$ However, the use of EtO carries the stigma of other risks, in that it is flammable, explosive and carcinogenic. Some modern EtO processes, such as that explored in this study, minimise these risks by utilising minute quantities $(<5 \mathrm{~g})$ of EtO per cycle, as well as various safeguards to ensure the operator receives negligible exposure to the gas. For additional safety, the system can be set inside a standard chemical fume hood. These considerations of risk should not be a barrier to adoption by the field.

This work marks the first study specifically exploring the effects of sterilisation on bio-inks for 3D bioprinting. We have limited the scope of this work to four common bio-ink components, however many dozens more remain to be explored. Regardless of the methods chosen, every sterilisation method will have some effect on bio-ink properties, and these effects need to be documented and discussed within the field.

\section{Acknowledgments}


Funding from the Australian Research Council Centre of Excellence Scheme (Project Number CE 140100012) is gratefully acknowledged. GelMA was obtained from the ANFF Materials Node.

\section{References}

1. Galante, R., Pinto, T. J. A. \& Serro, A. P. Review Article Sterilization of hydrogels for biomedical applications : A review. 1-21 (2017). doi:10.1002/jbm.b.34048

2. Di Foggia, M., Corda, U., Plescia, E., Taddei, P. \& Torreggiani, A. Effects of sterilisation by high-energy radiation on biomedical poly- $(\varepsilon-$ caprolactone)/hydroxyapatite composites. J. Mater. Sci. Mater. Med. 21, 1789-1797 (2010).

3. Hofmann, S., Stok, K. S., Kohler, T., Meinel, A. J. \& Müller, R. Effect of sterilization on structural and material properties of 3-D silk fibroin scaffolds. Acta Biomater. 10, 308-317 (2014).

4. Williams, D., Thayer, P., Martinez, H., Gatenholm, E. \& Khademhosseini, A. A perspective on the physical, mechanical and biological specifications of bioinks and the development of functional tissues in 3D bioprinting. Bioprinting 9, 19-36 (2018).

5. Ouyang, L., Yao, R., Zhao, Y. \& Sun, W. Effect of bioink properties on printability and cell viability for 3D bioplotting of embryonic stem cells. Biofabrication 8, 035020 (2016).

6. Li, Z. et al. Tuning Alginate-Gelatin Bioink Properties by Varying Solvent and Their Impact on Stem Cell Behavior. Sci. Rep. 8, 1-8 (2018).

7. Gao, T. et al. Optimization of gelatin-alginate composite bioink printability using rheological parameters: a systematic approach. Biofabrication 10, 034106 (2018).

8. Schuurman, W. et al. Gelatin-Methacrylamide Hydrogels as Potential Biomaterials for Fabrication of Tissue-Engineered Cartilage Constructs. Macromol. Biosci. 13, 551-61 (2013). 
9. He, Y. et al. Research on the printability of hydrogels in 3D bioprinting. Sci. Rep. 6, 29977 (2016).

10. Yue, K. et al. Synthesis, properties, and biomedical applications of gelatin methacryloyl (GelMA) hydrogels. Biomaterials 73, 254-271 (2015).

11. Nichol, J. W. et al. Cell-laden microengineered gelatin methacrylate hydrogels. Biomaterials 31, 5536-44 (2010).

12. O'Connell, C. D. et al. Tailoring the mechanical properties of gelatin methacryloyl hydrogels through manipulation of the photocrosslinking conditions. Soft Matter 14, 2142-2151 (2018).

13. Kang, H.-W. et al. A 3D bioprinting system to produce human-scale tissue constructs with structural integrity. Nat. Biotechnol. 34, 312-319 (2016).

14. Ding, C. Direct Bonding of Chitosan Biomaterials to Tissues Using Transglutaminase for Surgical Repair. 23, 135-142 (2017).

15. Jia, W. et al. Direct 3D bioprinting of perfusable vascular constructs using a blend bioink. Biomaterials 106, 58-68 (2016).

16. O'Connell, C. D. et al. Development of the Biopen: a handheld device for surgical printing of adipose stem cells at a chondral wound site. Biofabrication 8, 015019 (2016).

17. Loessner, D. et al. Functionalization, preparation and use of cell-laden gelatin methacryloyl-based hydrogels as modular tissue culture platforms. Nat. Protoc. 11, 727-746 (2016).

18. Yin, J., Yan, M., Wang, Y., Fu, J. \& Suo, H. 3D Bioprinting of Low-Concentration CellLaden Gelatin Methacrylate (GelMA) Bioinks with a Two-Step Cross-linking Strategy. ACS Appl. Mater. Interfaces 10, 6849-6857 (2018).

19. Bertassoni, L. E. et al. Direct-write bioprinting of cell-laden methacrylated gelatin hydrogels. Biofabrication 6, 024105 (2014).

20. Pepelanova, I., Kruppa, K., Scheper, T. \& Lavrentieva, A. Gelatin-Methacryloyl (GelMA) Hydrogels with Defined Degree of Functionalization as a Versatile Toolkit for 
3D Cell Culture and Extrusion Bioprinting. Bioengineering 5, 55 (2018).

21. Skardal, A. et al. A hydrogel bioink toolkit for mimicking native tissue biochemical and mechanical properties in bioprinted tissue constructs. Acta Biomater. 25, 24-34 (2015).

22. Bas, O. et al. Enhancing structural integrity of hydrogels by using highly organised melt electrospun fibre constructs. Eur. Polym. J. 72, 451-463 (2015).

23. Camci-Unal, G., Cuttica, D., Annabi, N., Demarchi, D. \& Khademhosseini, A. Synthesis and characterization of hybrid hyaluronic acid-gelatin hydrogels. Biomacromolecules 14, 1085-1092 (2013).

24. Duchi, S. et al. Handheld Co-Axial Bioprinting: Application to in situ surgical cartilage repair. Sci. Rep. 7, 5837 (2017).

25. Paxton, N. et al. Proposal to assess printability of bioinks for extrusion-based bioprinting and evaluation of rheological properties governing bioprintability. Biofabrication $\mathbf{9}$, (2017).

26. Armstrong, J. P. K., Burke, M., Carter, B. M., Davis, S. A. \& Perriman, A. W. 3D Bioprinting Using a Templated Porous Bioink. Adv. Healthc. Mater. 5, 1724-1730 (2016).

27. Müller, M., Öztürk, E., Arlov, Ø., Gatenholm, P. \& Zenobi-Wong, M. Alginate SulfateNanocellulose Bioinks for Cartilage Bioprinting Applications. Ann. Biomed. Eng. 45, 210-223 (2017).

28. Faramarzi, N. et al. Patient-Specific Bioinks for 3D Bioprinting of Tissue Engineering Scaffolds. Adv. Healthc. Mater. 7, 1-9 (2018).

29. Ahlfeld, T. et al. Development of a clay based bioink for 3D cell printing for skeletal application. Biofabrication 9, (2017).

30. Wu, Y., Lin, Z. Y. (William), Wenger, A. C., Tam, K. C. \& Tang, X. (Shirley). 3D bioprinting of liver-mimetic construct with alginate/cellulose nanocrystal hybrid bioink. Bioprinting 9, 1-6 (2018).

31. Li, Y. et al. 3D printing human induced pluripotent stem cells with novel hydroxypropyl 
chitin bioink: scalable expansion and uniform aggregation. Biofabrication 10, 044101 (2018).

32. Kang, H.-W., Yoo, J. J. \& Atala, A. Bioprinted Scaffolds for Cartilage Tissue Engineering. in Cartilage Tissue Engineering: Methods and Protocols (ed. Doran, P. M.) 161-169 (Springer New York, 2015). doi:10.1007/978-1-4939-2938-2_11

33. Burdick, J. A., Chung, C., Jia, X., Randolph, M. A. \& Langer, R. Controlled degradation and mechanical behavior of photopolymerized hyaluronic acid networks. Biomacromolecules 6, 386-91

34. GILBERT, G. L., GAMBILL, V. M., SPINER, D. R., HOFFMAN, R. K. \& PHILLIPS, C. R. EFFECT OF MOISTURE ON ETHYLENE OXIDE STERILIZATION. Appl. Microbiol. 12, 496-503 (1964).

35. Onofrillo, C. et al. Biofabrication of human articular cartilage: a path towards the development of a clinical treatment. Biofabrication 10, 045006 (2018).

36. US Food and Drug Administration. Guidance for Industry for Sterile Drug Products Produced by Aseptic Processing: Current Good Manufacturing Practice. Pharmaceutical CGMPs (2004).

37. Onyango, L. A., Dunstan, R. H. \& Roberts, T. K. Filterability of staphylococcal species through membrane filters following application of stressors. BMC Res. Notes 3, (2010).

38. Dai, Z., Ronholm, J., Tian, Y., Sethi, B. \& Cao, X. Sterilization techniques for biodegradable scaffolds in tissue engineering applications. J. Tissue Eng. 7, 204173141664881 (2016).

39. Mendes, G. C. C., Brandão, T. R. S. \& Silva, C. L. M. Ethylene oxide sterilization of medical devices: A review. Am. J. Infect. Control 35, 574-581 (2007).

40. Pedersen, M. R. \& Hansen, E. W. Inactivation of B. Subtilis Spores and E.Coli Endotoxin by Ethelene Oxide. J. Clin. Pharm. Ther. 14, 373-380 (1989).

41. Markstedt, K. et al. 3D bioprinting human chondrocytes with nanocellulose-alginate bioink for cartilage tissue engineering applications. Biomacromolecules 16, 1489-1496 (2015). 
42. Kesti, M., Fisch, P., Pensalfini, M., Mazza, E. \& Zenobi-Wong, M. Guidelines for standardization of bioprinting: A systematic study of process parameters and their effect on bioprinted structures. BioNanoMaterials 17, 193-204 (2016).

43. Van Den Bosch, E. \& Gielens, C. Gelatin degradation at elevated temperature. Int. J. Biol. Macromol. 32, 129-138 (2003).

44. Gantar, A., Sebenik, A., Kavbi, M. \& Osredkar, U. Study of grafting ethylene oxide onto gelatin by one- and hetero two-dimensional 13C n . m . r, spectroscopy. 28, 1403-1406 (1987).

45. Bothner, H., Waaler, T. \& Wik, O. Limiting viscosity number and weight average molecular weight of hyaluronate samples produced by heat degradation. Int. J. Biol. Macromol. 10, 287-291 (1988).

46. Reháková, M., Bakoš, D., Soldán, M. \& Vizárová, K. Depolymerization reactions of hyaluronic acid in solution. Int. J. Biol. Macromol. 16, 121-124 (1994).

47. Lundin, A., Panas, I. \& Ahlberg, E. A mechanistic investigation of ethylene oxide hydrolysis to ethanediol. J. Phys. Chem. A 111, 9087-9092 (2007).

48. Parsons, B. J., Al-Assaf, S., Navaratnam, S. \& Phillips, G. O. COMPARISON OF THE REACTIVITY OF DIFFERENT REACTIVE OXIDATIVE SPECIES (ROS) TOWARDS HYALURONAN. in Hyaluronan 141-150 (Elsevier, 2002). doi:10.1533/9781845693121.151

49. Kvam, C., Granese, D., Flaibani, A., Pollesello, P. \& Paoletti, S. Hyaluronan Can Be Protected from Free-Radical Depolymerization by 2,6-Diisopropylphenol, a Novel Radical Scavenger. Biochem. Biophys. Res. Commun. 193, 927-933 (1993).

50. Pierce, B. F. et al. Photocrosslinked Co-Networks from Glycidylmethacrylated Gelatin and Poly(ethylene glycol) Methacrylates. Macromol. Biosci. 12, 484-493 (2012).

51. Windmueller, H. G., Ackerman, C. J. \& Engel, R. W. Reaction of Ethylene Oxide with Histidine, Methionine, and Cysteine. J. Biol. Chem. 234, 895-899 (1959).

52. Yeung, C.-Y. et al. Negative effect of heat sterilization on the free amino acid concentrations in infant formula. Eur. J. Clin. Nutr. 60, 136-141 (2006). 
53. Kyle, S., Jessop, Z. M., Al-Sabah, A. \& Whitaker, I. S. 'Printability' of Candidate Biomaterials for Extrusion Based 3D Printing: State-of-the-Art. Adv. Healthc. Mater. 1700264, 1-16 (2017).

54. Vink, P. \& Pleijsier, K. Aeration of ethylene oxide-sterilized polymers. Biomaterials 7, 225-230 (1986).

55. ISO 11135:2014: Sterilization of health-care products -- Ethylene oxide -- Requirements for the development, validation and routine control of a sterilization process for medical devices. (2014).

56. ISO 10993-7:2008 Biological evaluation of medical devices -- Part 7: Ethylene oxide sterilization residuals.

57. Mochida, K. \& Gomyoda, M. Toxicity of ethylene glycol, diethylene glycol, and propylene glycol to human cells in culture. Bull. Environ. Contam. Toxicol. 38, 151153 (1987).

58. Barbucci, R. et al. Hyaluronic acid hydrogel in the treatment of osteoarthritis. Biomaterials 23, 4503-4513 (2002).

59. Marreco, P. R., Moreira, P. da L., Genari, S. C. \& Moraes, 2 ngela Maria. Effects of different sterilization methods on the morphology, mechanical properties, and cytotoxicity of chitosan membranes used as wound dressings. J. Biomed. Mater. Res. 71B, 268-277 (2004).

60. Hastings, C. E. et al. The effects of ethylene oxide sterilization on the in vitro cytotoxicity of a bone replacement material. Toxicol. Vitr. 4, 757-762 (1990).

61. Ponticiello, M. S., Schinagl, R. M., Kadiyala, S. \& Barry, F. P. Gelatin-based resorbable sponge as a carrier matrix for human mesenchymal stem cells in cartilage regeneration therapy. J. Biomed. Mater. Res. 52, 246-255 (2000).

62. Tan, H., Wu, J., Lao, L. \& Gao, C. Gelatin/chitosan/hyaluronan scaffold integrated with PLGA microspheres for cartilage tissue engineering. Acta Biomater. 5, 328-337 (2009).

63. Deng, T., Huang, S., Zhou, S., He, L. \& Jin, Y. Cartilage regeneration using a novel gelatin-chondroitin-hyaluronan hybrid scaffold containing bFGF-impregnated 
microspheres. J. Microencapsul. 24, 163-174 (2007).

64. Di Bella, C. et al. In situ handheld three-dimensional bioprinting for cartilage regeneration. J. Tissue Eng. Regen. Med. 1-11 (2017). doi:10.1002/term.2476

65. Levett, P. A. et al. Photocrosslinkable hydrogels for cartilage tissue engineering. Journal of Tissue Engineering and Regenerative Medicine 6, (2012).

66. Levett, P. A. et al. A biomimetic extracellular matrix for cartilage tissue engineering centered on photocurable gelatin, hyaluronic acid and chondroitin sulfate. Acta Biomater. 10, 214-223 (2014).

67. Levett, P. a., Hutmacher, D. W., Malda, J. \& Klein, T. J. Hyaluronic Acid Enhances the Mechanical Properties of Tissue-Engineered Cartilage Constructs. PLoS One 9, e113216 (2014).

68. Toh, W. S. et al. Cartilage repair using hyaluronan hydrogel-encapsulated human embryonic stem cell-derived chondrogenic cells. Biomaterials 31, 6968-6980 (2010).

69. Muzzarelli, R. A. A., Greco, F., Busilacchi, A., Sollazzo, V. \& Gigante, A. Chitosan, hyaluronan and chondroitin sulfate in tissue engineering for cartilage regeneration: A review. Carbohydr. Polym. 89, 723-739 (2012).

70. Kim, I. L., Mauck, R. L. \& Burdick, J. A. Hydrogel design for cartilage tissue engineering: A case study with hyaluronic acid. Biomaterials 32, 8771-8782 (2011).

71. Toh, W. S., Lim, T. C., Kurisawa, M. \& Spector, M. Modulation of mesenchymal stem cell chondrogenesis in a tunable hyaluronic acid hydrogel microenvironment. Biomaterials 33, 3835-3845 (2012).

72. Ye, K. et al. Chondrogenesis of Infrapatellar Fat Pad Derived Adipose Stem Cells in 3D Printed Chitosan Scaffold. PLoS One 9, e99410 (2014).

73. Sophia Fox, A. J., Bedi, A. \& Rodeo, S. A. The basic science of articular cartilage: structure, composition, and function. Sports Health 1, 461-8 (2009).

74. Lefebvre, V., Huang, W., Harley, V. R., Goodfellow, P. N. \& de Crombrugghe, B. SOX9 is a potent activator of the chondrocyte-specific enhancer of the pro alpha1(II) 
collagen gene. Mol. Cell. Biol. 17, 2336-2346 (1997).

75. Mansour, J. M. \& Ph, D. Biomechanics of Cartilage. in Kinesiology: the mechanics and pathomechanics of human movement 66-79 (2009).

76. Hodgetts, S. I., Beilharz, M. W., Scalzo, A. A. \& Grounds, M. D. Why Do Cultured Transplanted Myoblasts Die in Vivo? DNA Quantification Shows Enhanced Survival of Donor Male Myoblasts in Host Mice Depleted of CD4 + and CD8 + Cells or NK1.1 + Cells. Cell Transplant. 9, 489-502 (2000).

77. Levato, R. et al. The bio in the ink: cartilage regeneration with bioprintable hydrogels and articular cartilage-derived progenitor cells. Acta Biomater. 61, 41-53 (2017).

78. Christopher, M. O., Francoise, M., Gabor, F. \& Cheryl, M. H. Biofabrication and testing of a fully cellular nerve graft. Biofabrication 5, 45007 (2013).

79. Berner, A. et al. Scaffold-cell bone engineering in a validated preclinical animal model: precursors vs differentiated cell source. J. Tissue Eng. Regen. Med. 11, 2081-2089 (2017).

80. Randall, M. J., Jüngel, A., Rimann, M. \& Wuertz-Kozak, K. Advances in the Biofabrication of 3D Skin in vitro: Healthy and Pathological Models. Front. Bioeng. Biotechnol. 6, (2018). 